\title{
AN EXTENSION OF THE MASKIT SLICE FOR 4-DIMENSIONAL KLEINIAN GROUPS
}

\author{
YOSHIAKI ARAKI AND KENTARO ITO
}

\begin{abstract}
Let $\Gamma$ be a 3-dimensional Kleinian punctured torus group with accidental parabolic transformations. The deformation space of $\Gamma$ in the group of Möbius transformations on the 2-sphere is well known as the Maskit slice $\mathcal{M}_{1,1}$ of punctured torus groups. In this paper, we study deformations $\Gamma^{\prime}$ of $\Gamma$ in the group of Möbius transformations on the 3-sphere such that $\Gamma^{\prime}$ does not contain screw parabolic transformations. We will show that the space of the deformations is realized as a domain of 3-space $\mathbb{R}^{3}$, which contains the Maskit slice $\mathcal{M}_{1,1}$ as a slice through a plane. Furthermore, we will show that the space also contains the Maskit slice $\mathcal{M}_{0,4}$ of fourth-punctured sphere groups as a slice through another plane. Some of the other slices of the space will be also studied.
\end{abstract}

\section{INTRODUCTION}

Let $\Gamma$ be a 3 -dimensional Kleinian once-punctured (or simply punctured) torus group with accidental parabolic transformations, which acts on the Riemann sphere $\hat{\mathbb{C}}$ as Möbius transformations. In this paper, we regard $\Gamma$ as a 4-dimensional Kleinian group and study deformations $\Gamma^{\prime}$ of $\Gamma$ in the group of Möbius transformations on the 3 -sphere $\hat{\mathbb{R}}^{3}=\mathbb{R}^{3} \cup\{\infty\}$; a Kleinian group $\Gamma^{\prime}$ is called a deformation of $\Gamma$ if there is an isomorphism $\phi: \Gamma \rightarrow \Gamma^{\prime}$ which takes a parabolic transformation to a parabolic transformation. Especially, we focus on all deformations $\left\{\phi: \Gamma \rightarrow \Gamma^{\prime}\right\}$ of $\Gamma$ such that $\phi$ takes a pure parabolic transformation to a pure parabolic transformation; the definition of a pure/screw parabolic transformation will be described below (see also 2.5).

The $n$-dimensional sphere $\hat{\mathbb{R}}^{n}=\mathbb{R}^{n} \cup\{\infty\}$ is naturally identified with the ideal boundary of the $(n+1)$-dimensional hyperbolic space $\mathbf{H}^{n+1}$. Therefore, the group $\operatorname{Möb}\left(\hat{\mathbb{R}}^{n}\right)$ of orientation preserving Möbius transformations of $\hat{\mathbb{R}}^{n}$ is identified with the group Isom $\left(\mathbf{H}^{n+1}\right)$ of orientation preserving isomorphisms of $\mathbf{H}^{n+1}$. A discrete subgroup of $\operatorname{Isom}\left(\mathbf{H}^{n+1}\right)=\operatorname{Möb}\left(\hat{\mathbb{R}}^{n}\right)$ is said to be an $(n+1)$-dimensional Kleinian group.

3-dimensional Kleinian groups are well studied in various contexts; for example, hyperbolic geometry, 3-dimensional topology, complex analysis, etc. We refer the reader to Kapovich 8 and Marden 11 for overviews of the theory of 3-dimensional Kleinian groups. On the other hand, the study of Kleinian groups in higher dimensions is more wild and is far from getting the whole picture. We refer the reader to Apanasov [2 and Kapovich 7] for more information in this area. Among higher dimensional Kleinian groups, the study of 4-dimensional Kleinian groups should be

Received by the editors April 1, 2008.

(C)2008 American Mathematical Society Reverts to public domain 28 years from publication 
interesting in its own light because they have "fractal" limit sets in the 3-sphere $\hat{\mathbb{R}}^{3}$ into which our geometric insight work well. Some examples of 4-dimensional Kleinian groups with computer graphics of their limit sets can be found in AharaAraki [1].

We now move into a more detailed argument. In what follows, we regard $\operatorname{Möb}\left(\hat{\mathbb{R}}^{2}\right)$ as a subgroup of $\operatorname{Möb}\left(\hat{\mathbb{R}}^{3}\right)$ by embedding $\hat{\mathbb{R}}^{2}$ into $\hat{\mathbb{R}}^{3}$ by $(x, y) \mapsto(x, y, 0)$. Recall that the group $\operatorname{Möb}\left(\hat{\mathbb{R}}^{2}\right)$ is naturally identified with the group $\operatorname{Aut}(\hat{\mathbb{C}})$ of conformal automorphisms of the Riemann sphere $\hat{\mathbb{C}}$, or the group of linear fractional transformations. Let $\Gamma \subset \operatorname{Möb}\left(\hat{\mathbb{R}}^{2}\right)$ be a Kleinian punctured torus group with accidental parabolic transformations; that is, $\Gamma$ is a 3 -dimensional Kleinian group such that the quotient manifold $\mathbf{H}^{3} / \Gamma$ is homeomorphic to the trivial interval bundle over a punctured torus, and the ideal conformal boundary of $\mathbf{H}^{3} / \Gamma$ is a union of a punctured torus and a thrice-punctured sphere. Then $\Gamma$ is a rank- 2 free group and a pair of generators $\alpha, \beta \in \operatorname{Mob}\left(\hat{\mathbb{R}}^{2}\right)$ of $\Gamma$ can be chosen so that $\beta$ and the commutator $[\alpha, \beta]$ of the generators are parabolic. The following normalization of such a group $\Gamma$ up to conjugation is fundamental.

Lemma 1.1 (cf. 9] and [10). Let $\Gamma=\langle\alpha, \beta\rangle$ be a (not necessarily discrete) rank-2 free group in $\mathrm{Möb}\left(\hat{\mathbb{R}}^{2}\right)$ such that $\beta$ and $[\alpha, \beta]$ are parabolic. Then there is a complex number $\mu \in \mathbb{C}$ such that $\Gamma$ is conjugate in $\operatorname{Mob}\left(\hat{\mathbb{R}}^{2}\right)$ to the group $G_{\mu}=\left\langle A_{\mu}, B\right\rangle$ generated by two Möbius transformations

$$
A_{\mu}(\tau)=\frac{1}{\tau}+\mu \quad \text { and } \quad B(\tau)=\tau+2
$$

We define:

$$
\mathcal{M}_{1,1}=\left\{\mu \in \mathbb{C}: G_{\mu}=\left\langle A_{\mu}, B\right\rangle \text { is a rank-2 free Kleinian group }\right\} .
$$

This set is known as the Maskit slice of 3-dimensional Kleinian punctured torus groups (see [10] for more information).

In this paper we want to consider deformations of a 3-dimensional Kleinian group $G_{\mu}=\left\langle A_{\mu}, B\right\rangle$ with $\mu \in \mathcal{M}_{1,1}$ in the group $\operatorname{Möb}\left(\hat{\mathbb{R}}^{3}\right)$. To explain our results, we need to recall a classification of elements of $\operatorname{Mob}\left(\hat{\mathbb{R}}^{3}\right)$; see Section 2 for more details. Similar to the case of $\operatorname{Möb}\left(\hat{\mathbb{R}}^{2}\right)$, elements of $\operatorname{Möb}\left(\hat{\mathbb{R}}^{3}\right)$ are classified into three types: elliptic, parabolic and loxodromic transformations. Especially a transformation in $\operatorname{Möb}\left(\hat{\mathbb{R}}^{3}\right)$ is parabolic if it has exactly one fixed point in $\hat{\mathbb{R}}^{3}$. Those parabolic elements are further classified into two types: a parabolic transformation $f \in \operatorname{Möb}\left(\hat{\mathbb{R}}^{3}\right)$ is said to be pure parabolic if it is conjugate in $\operatorname{Mob}\left(\hat{\mathbb{R}}^{3}\right)$ to a translation, and screw parabolic otherwise. A screw parabolic transformation is conjugate to a composition of a rotation and a translation along a common axis.

The first goal of this paper is to show the following theorem which is an analogue of Lemma 1.1 for groups in $\operatorname{Möb}\left(\hat{\mathbb{R}}^{3}\right)$ :

Theorem 1.2 (Theorem 4.5). Let $\Gamma=\langle\alpha, \beta\rangle$ be a (not necessarily discrete) rank2 free group in $\operatorname{Möb}\left(\hat{\mathbb{R}}^{3}\right)$ such that $\beta$ and $[\alpha, \beta]$ are pure parabolic. Then there is a point $\boldsymbol{p}=(p, q, r)$ in $\mathbb{R}^{3}$ such that $\Gamma$ is conjugate in $\mathrm{Möb}\left(\hat{\mathbb{R}}^{3}\right)$ to the group $G_{\boldsymbol{p}}=\left\langle A_{\boldsymbol{p}}, B\right\rangle$ generated by

$$
A_{p}(x, y, z)=\frac{(x,-y, z)}{x^{2}+y^{2}+z^{2}}+(p, q, r)
$$


and

$$
B(x, y, z)=(x, y, z)+(2,0,0) .
$$

It is just a technical reason why we assume in the theorem above that $\beta$ and $[\alpha, \beta]$ are "pure parabolic" instead of "parabolic" and it is more natural to consider the case where $\beta$ and $[\alpha, \beta]$ could be screw parabolic. In fact, suppose that a homeomorphism $f: \hat{\mathbb{R}}^{3} \rightarrow \hat{\mathbb{R}}^{3}$ conjugates a 3 -dimensional Kleinian group $\Gamma \subset \operatorname{Möb}\left(\hat{\mathbb{R}}^{2}\right)$ with a parabolic transformation $\gamma \in \Gamma$ to a 4-dimensional Kleinian group $\Gamma^{\prime}=f \Gamma f^{-1} \subset \operatorname{Möb}\left(\hat{\mathbb{R}}^{3}\right)$. Then the transformation $f \gamma f^{-1} \in \Gamma^{\prime}$ could be screw parabolic in general (see 2.5). Unfortunately, we do not know the result of Theorem 1.2 with "pure parabolic" replaced by "parabolic." We also remark that, recently, Y. Kim and K. Sakugawa independently announced that the level 2 congruence subgroup of the modular group, the rank-2 free group $\Gamma=\langle\alpha, \beta\rangle$ in $\operatorname{Möb}\left(\hat{\mathbb{R}}^{2}\right)$ such that $\alpha, \beta$ and $\alpha \beta$ are parabolic, has a continuous family of deformations $\left\{\Gamma^{\prime}\right\}$ in $\operatorname{Möb}\left(\hat{\mathbb{R}}^{3}\right)$ such that $\Gamma^{\prime}$ are Kleinian groups containing screw parabolic transformations (see also Theorem 4.2 and its remark).

We define:

$$
\widehat{\mathcal{M}}_{1,1}=\left\{\boldsymbol{p}=(p, q, r) \in \mathbb{R}^{3}: G_{\boldsymbol{p}}=\left\langle A_{\boldsymbol{p}}, B\right\rangle \text { is a rank-2 free Kleinian group }\right\} .
$$

The next purpose of this paper is to understand the shape of the set $\widehat{\mathcal{M}}_{1,1}$. We remark that if $\boldsymbol{p}=(p, q, 0)$, the group $G_{\boldsymbol{p}}=\left\langle A_{\boldsymbol{p}}, B\right\rangle$ is nothing but the Poincaré extension of the group $G_{\mu}=\left\langle A_{\mu}, B\right\rangle$ with $\mu=p+i q$. Therefore $\widehat{\mathcal{M}}_{1,1}$ contains $\mathcal{M}_{1,1}$ as a slice through the plane $r=0$ in the parameter space $\mathbb{R}^{3}=\{\boldsymbol{p}=(p, q, r)\}$. On the other hand, we will show in Section 5 that the slice of $\widehat{\mathcal{M}}_{1,1}$ through the plane $q=0$ is the Maskit slice $\mathcal{M}_{0,4}$ of 3 -dimensional fourth-punctured sphere groups which is defined by

$$
\mathcal{M}_{0,4}=\left\{\mu \in \mathbb{C}: H_{\mu}=\left\langle B, C, D_{\mu}\right\rangle \text { is a rank-3 free Kleinian group }\right\},
$$

where $B(\tau)=\tau+2, C(\tau)=\tau /(2 \tau+1)$ and $D_{\mu}(\tau)=C(\tau-\mu)+\mu$ (see Section 3).

Theorem 1.3 (Theorems 5.4 and 5.7). The Maskit slice $\mathcal{M}_{0,4}$ appears as the slice of $\widehat{\mathcal{M}}_{1,1}$ through the plane $q=0$. Moreover, there is a constant $0<\varphi_{0}<\pi / 2$ such that for every $\theta \in\left[-\varphi_{0}, \varphi_{0}\right], \mathcal{M}_{0,4}$ also appears as the slice through the plane $q=0$ with angle $\theta$ rotated along the p-axis.

Although the boundary of the slices of $\widehat{\mathcal{M}}_{1,1}$ through the planes $r=0$ and $q=0$ are so called "fractal," we will also obtain in Section 5 the following theorem (see also Figure 1).

Theorem 1.4 (Corollary 5.11). The boundary of the slice of $\widehat{\mathcal{M}}_{1,1}$ through the plane $p=0$ is a union of countably many analytic arcs.

One can find in Figure 2 some computer graphics of the limit sets of the groups $G_{\boldsymbol{p}}$ for parameters $\boldsymbol{p} \in \mathbb{R}^{3}$ which lie (or seem to lie) in the set $\widehat{\mathcal{M}}_{1,1}$. These figures can be seen as 3-dimensional extensions of the beautiful patterns in the complex plane included in the book by Mumford, Series and Wright [15].

We remark that Möbius transformations in $\operatorname{Möb}\left(\hat{\mathbb{R}}^{3}\right)$ can be related to two-bytwo matrices whose entries lie in the quaternion algebra; see Cao-Parker-Wang [5]. However, in this paper, we take a geometric approach and make use of geometric techniques in the theory of Kleinian groups, instead of calculating in the quaternion algebra. 


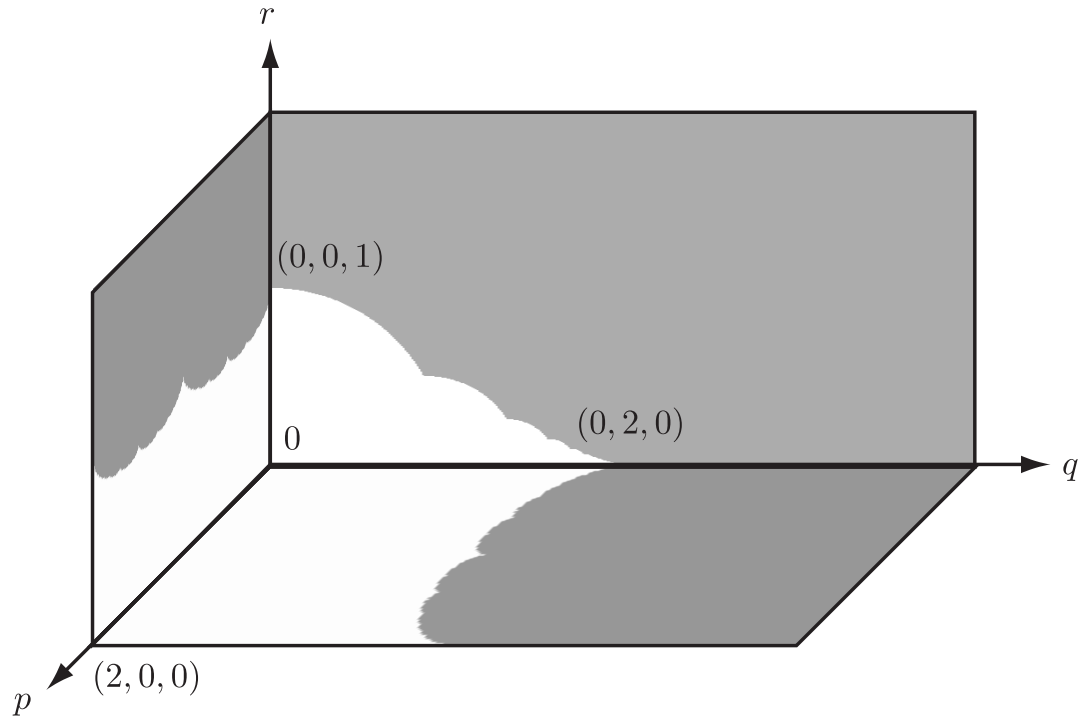

Figure 1. A schematic figure of slices of $\widehat{\mathcal{M}}_{1,1}$ through the planes $p=0, q=0$ and $r=0$.

This paper is organized as follows: In Section 2, we recall some basic facts of Möbius transformations. In Section 3, we recall the definition of the Maskit slice $\mathcal{M}_{1,1}$ (resp. $\mathcal{M}_{0,4}$ ) of 3-dimensional punctured torus groups (resp. fourthpunctured sphere groups). In Section 4, we prove Theorem 1.2 on a normalization of 4-dimensional punctured torus groups with accidental parabolics. In Section 5, we study the shape of the space $\widehat{\mathcal{M}}_{1,1}$ and prove Theorems 1.3 and 1.4. As an application of Theorem 1.2, we give in Section 6, Appendix, an example of a family of 4-dimensional Kleinian groups with 3-generators, which contain punctured torus groups as 2-generator subgroups.

\section{Preliminaries}

In this section, we recall from Beardon [4] and Matsumoto [13] the basic facts of Möbius transformations on an $n$-dimensional sphere $\hat{\mathbb{R}}^{n}=\mathbb{R}^{n} \cup\{\infty\}$. In the succeeding sections, we are mainly concerned with the cases of $n=2,3$.

2.1. Inversions. For $\boldsymbol{x}=\left(x_{1}, \ldots, x_{n}\right) \in \mathbb{R}^{n}$,

$$
|\boldsymbol{x}|=\sqrt{x_{1}^{2}+\cdots+x_{n}^{2}}
$$

denotes the Euclidean norm of $\boldsymbol{x}$. An $(n-1)$-dimensional sphere $\sigma$ in $\hat{\mathbb{R}}^{n}$ is either an Euclidean sphere $\left\{\boldsymbol{x} \in \mathbb{R}^{n}:|\boldsymbol{x}-\boldsymbol{a}|=r\right\}$ with $\boldsymbol{a} \in \mathbb{R}^{n}, r>0$, or an $(n-1)$ dimensional Euclidean plane $P$ plus $\{\infty\}$. The inversion $J_{\sigma}: \hat{\mathbb{R}}^{n} \rightarrow \hat{\mathbb{R}}^{n}$ in the sphere $\sigma$ is defined as follows: if $\sigma=\left\{\boldsymbol{x} \in \mathbb{R}^{n}:|\boldsymbol{x}-\boldsymbol{a}|=r\right\}$, then

$$
J_{\sigma}(\boldsymbol{x})=r^{2} \frac{\boldsymbol{x}-\boldsymbol{a}}{|\boldsymbol{x}-\boldsymbol{a}|^{2}}+\boldsymbol{a},
$$




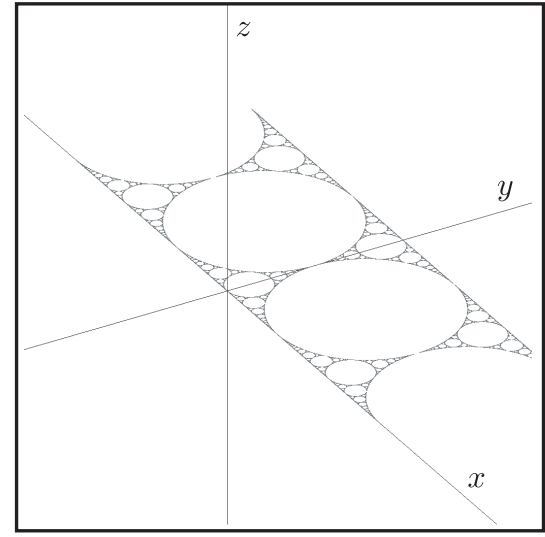

$(p, q, r)=(0,2,0)$

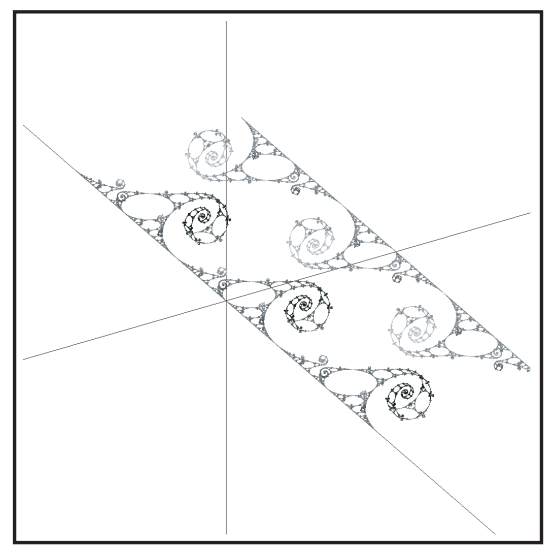

$(p, q, r)=(0,1.9,0.04)$

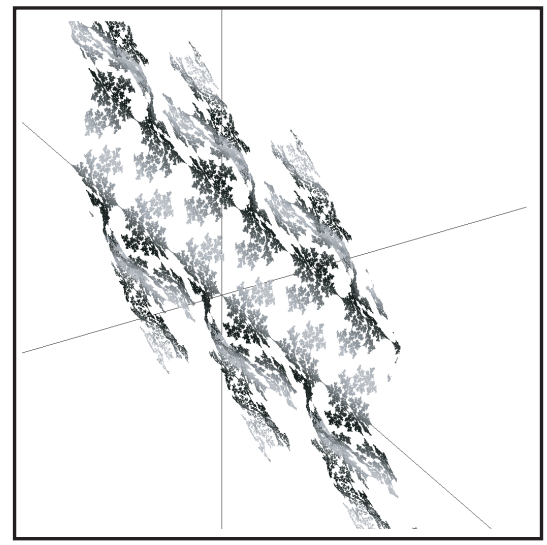

$(p, q, r)=(-0.2,0.3,0.8)$

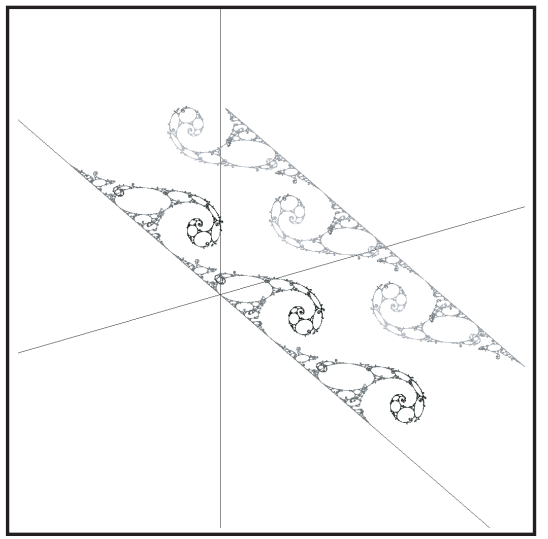

$(p, q, r)=(-0.1,1.8,0.1)$

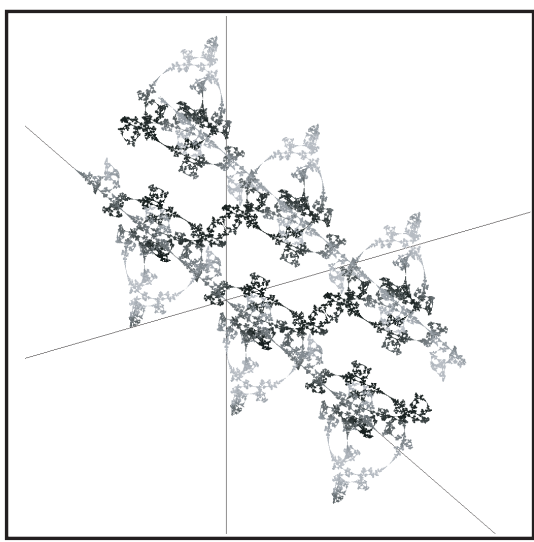

$(p, q, r)=(0.2,1,0.5)$

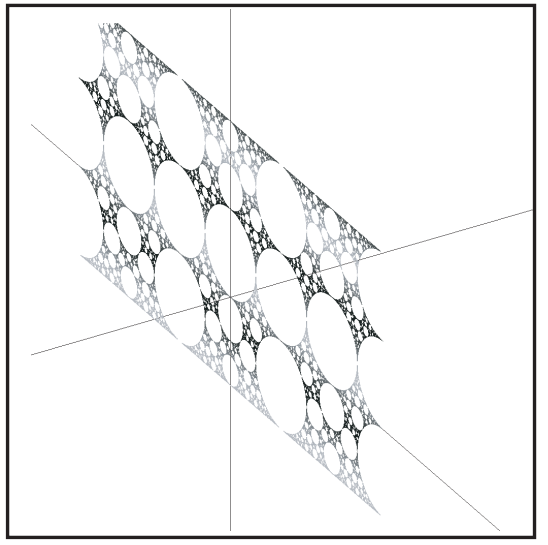

$(p, q, r)=(0,0,1)$

Figure 2. Computer graphics of the limit sets $\Lambda\left(G_{\boldsymbol{p}}\right)$ of $G_{\boldsymbol{p}}$ restricted to the domain $\left\{(x, y, z) \in \mathbb{R}^{3}:|x| \leq 3\right\}$. The brightness of a point $(x, y, z) \in \Lambda\left(G_{\boldsymbol{p}}\right)$ depends on its $z$-value. These are the views from the same point. 
and if $\sigma=P \cup\{\infty\}$, then $J_{\sigma}$ is the reflection in the plane $P$. In particular, we denote the inversion in the unit sphere at the origin by

$$
J(\boldsymbol{x})=\frac{\boldsymbol{x}}{|\boldsymbol{x}|^{2}} .
$$

Then the inversion $J_{\sigma}$ in (2.1) is written as

$$
J_{\sigma}(\boldsymbol{x})=r^{2} J(\boldsymbol{x}-\boldsymbol{a})+\boldsymbol{a} .
$$

2.2. Möbius transformations. A Möbius transformation on $\hat{\mathbb{R}}^{n}$ is a product of finite numbers of inversions in codimension one spheres in $\hat{\mathbb{R}}^{n}$. We denote by $\operatorname{Möb}\left(\hat{\mathbb{R}}^{n}\right)$ the group of orientation preserving Möbius transformations. It is known that the group $\operatorname{Möb}\left(\hat{\mathbb{R}}^{n}\right)$ is equal to the group of all orientation preserving conformal automorphism of $\hat{\mathbb{R}}^{n}$. In particular, the group $\operatorname{Möb}\left(\hat{\mathbb{R}}^{2}\right)$ is naturally identified with the group Aut $(\hat{\mathbb{C}})$ of orientation preserving conformal automorphism of $\hat{\mathbb{C}}$ and with the Lie group $\mathrm{PSL}_{2}(\mathbb{C})$. The followings are typical examples of transformations $f: \hat{\mathbb{R}}^{n} \rightarrow \hat{\mathbb{R}}^{n}$ in $\operatorname{Möb}\left(\hat{\mathbb{R}}^{n}\right):$

- A translation: $f(\boldsymbol{x})=\boldsymbol{x}+\boldsymbol{a}$, where $\boldsymbol{a} \in \mathbb{R}^{n}$. We denote by $T_{\boldsymbol{a}}(\boldsymbol{x})=\boldsymbol{x}+\boldsymbol{a}$.

- A magnification: $f(\boldsymbol{x})=\lambda \boldsymbol{x}$, where $\lambda>0$.

- An orthonormal transformation: $f(\boldsymbol{x})=P(\boldsymbol{x})$, where $P \in S O(n)$.

General Möbius transformations can be written as follows.

Lemma 2.1 (Theorem 3.5.1 in प4). Let $f \in \operatorname{Möb}\left(\hat{\mathbb{R}}^{n}\right)$.

(1) If $f(\infty)=\infty$, then $f(\boldsymbol{x})=\lambda P(\boldsymbol{x})+\boldsymbol{u}$, where $\lambda>0, P \in S O(n)$ and $\boldsymbol{u} \in \mathbb{R}^{n}$.

(2) If $f(\infty) \neq \infty$, then $f(\boldsymbol{x})=\lambda P J(\boldsymbol{x}-\boldsymbol{u})+\boldsymbol{v}$, where $\lambda>0, P \in O(n) \backslash S O(n)$ and $\boldsymbol{u}, \boldsymbol{v} \in \mathbb{R}^{n}$.

2.3. Isometric spheres. Let $f \in \operatorname{Möb}\left(\hat{\mathbb{R}}^{n}\right)$ be the same as in Lemma 2.1(2). Note that $\boldsymbol{u}=f^{-1}(\infty)$ and $\boldsymbol{v}=f(\infty)$. The isometric sphere $I(f)$ of $f$ is defined by

$$
I(f)=\left\{\boldsymbol{x} \in \mathbb{R}^{n}:|\boldsymbol{x}-\boldsymbol{u}|=\sqrt{\lambda}\right\} .
$$

It follows from the equality (2.2) that the inversion in $I(f)$ is written as $J_{I(f)}(\boldsymbol{x})=$ $\lambda J(\boldsymbol{x}-\boldsymbol{u})+\boldsymbol{u}$. Thus the transformation $f(\boldsymbol{x})=\lambda P J(\boldsymbol{x}-\boldsymbol{u})+\boldsymbol{v}$ can be written as

$$
f(\boldsymbol{x})=P\left(J_{I(f)}(\boldsymbol{x})-\boldsymbol{u}\right)+\boldsymbol{v} .
$$

From this, one can observe that $f$ maps the interior of $I(f)=\left\{\boldsymbol{x} \in \mathbb{R}^{n}:|\boldsymbol{x}-\boldsymbol{u}|=\right.$ $\sqrt{\lambda}\}$ onto the exterior of $I\left(f^{-1}\right)=\left\{\boldsymbol{x} \in \mathbb{R}^{n}:|\boldsymbol{x}-\boldsymbol{v}|=\sqrt{\lambda}\right\}$. In particular, the map $f$ restricted to $I(f)$ is an isometry.

2.4. Poincaré extensions. We embed $\hat{\mathbb{R}}^{n}$ in $\hat{\mathbb{R}}^{n+1}$ in the natural way by $\left(x_{1}, \ldots, x_{n}\right) \in \mathbb{R}^{n} \mapsto\left(x_{1}, \ldots, x_{n}, 0\right) \in \mathbb{R}^{n+1}$ and $\infty \mapsto \infty$. Then for every $f \in \operatorname{Möb}\left(\hat{\mathbb{R}}^{n}\right)$, there is a unique $\tilde{f} \in \operatorname{Möb}\left(\hat{\mathbb{R}}^{n+1}\right)$, called the Poincaré extension of $f$, such that $\left.\tilde{f}\right|_{\hat{\mathbb{R}}^{n}}=f$. In fact, if $f$ is a composition of inversions in $(n-1)$ dimensional spheres $\sigma_{1}, \ldots, \sigma_{k}$ in $\hat{\mathbb{R}}^{n}, \tilde{f}$ is obtained by the composition of inversions in $n$-dimensional spheres $\tilde{\sigma}_{1}, \ldots, \tilde{\sigma}_{k}$ in $\hat{\mathbb{R}}^{n+1}$, where $\tilde{\sigma}_{i}$ is the sphere which is orthogonal to $\hat{\mathbb{R}}^{n}$ at $\sigma_{i}$. In this way, $\operatorname{Möb}\left(\hat{\mathbb{R}}^{n}\right)$ can be regarded as a subgroup of $\operatorname{Möb}\left(\hat{\mathbb{R}}^{n+1}\right)$. Let

$$
\mathbf{H}^{n+1}=\left\{\left(x_{1}, \ldots, x_{n+1}\right) \in \mathbb{R}^{n+1}: x_{n+1}>0\right\}
$$


denote the upper half-space of $\hat{\mathbb{R}}^{n+1}$ equipped with the hyperbolic metric $d s^{2}=$ $\left(d x_{1}^{2}+\cdots+d x_{n+1}^{2}\right) / x_{n+1}^{2}$. It is the upper half-space model of the $(n+1)$-dimensional hyperbolic space. For every $f \in \operatorname{Möb}\left(\hat{\mathbb{R}}^{n}\right)$, its Poincaré extension $\tilde{f} \in \operatorname{Möb}\left(\hat{\mathbb{R}}^{n+1}\right)$ induces an orientation preserving isomorphism of $\mathbf{H}^{n+1}$. In this way, $\operatorname{Möb}\left(\hat{\mathbb{R}}^{n}\right)$ can be identified with the group $\operatorname{Isom}\left(\mathbf{H}^{n+1}\right)$ of orientation preserving isomorphism of $\mathbf{H}^{n+1}$.

\subsection{Classifications of Möbius transformations.}

Definition 2.2. Let $f \in \operatorname{Möb}\left(\hat{\mathbb{R}}^{n}\right)=\operatorname{Isom}\left(\mathbf{H}^{n+1}\right)$. We say that $f$ is elliptic if $f$ has a fixed point in $\mathbf{H}^{n+1}$. If $f$ is not elliptic, $f$ is said to be parabolic if $f$ has exactly one fixed point in $\hat{\mathbb{R}}^{n}$, and loxodromic if $f$ has exactly two fixed points in $\hat{\mathbb{R}}^{n}$.

It is known that every element $f \in \operatorname{Möb}\left(\hat{\mathbb{R}}^{n}\right)$ is either elliptic, parabolic or loxodromic (see (2.23) in [13]). To describe a standard form of elliptic transformations, it is convenient to consider in the unit ball model $\mathbf{B}^{n+1}=\left\{\boldsymbol{x} \in \mathbb{R}^{n+1}:|\boldsymbol{x}|<1\right\}$ of the hyperbolic space: In this setting, if $f \in \operatorname{Isom}\left(\mathbf{B}^{n+1}\right)$ is elliptic with $f(\mathbf{0})=\mathbf{0}$, then $f(\boldsymbol{x})=P(\boldsymbol{x})$ for some $P \in S O(n+1)$. In the rest of the paper, we always consider in the upper half-space model of the hyperbolic space. Standard forms of parabolic and loxodromic transformations are given in the following:

Lemma 2.3 ((2.24) in [13]). Let $f \in \operatorname{Möb}\left(\hat{\mathbb{R}}^{n}\right)$.

(1) If $f$ is a parabolic transformation with a fixed point $\infty$, then $f(\boldsymbol{x})=P(\boldsymbol{x})+$ $\boldsymbol{u}$ for some $\boldsymbol{u} \in \mathbb{R}^{n} \backslash\{\mathbf{0}\}$ and $P \in S O(n)$ with $P(\boldsymbol{u})=\boldsymbol{u}$.

(2) If $f$ is a loxodromic transformation with fixed points $\mathbf{0}, \infty$, then $f(\boldsymbol{x})=$ $\lambda P(\boldsymbol{x})$ for some $\lambda>0$ with $\lambda \neq 1$ and $P \in S O(n)$.

Definition 2.4 (pure parabolic and screw parabolic). A parabolic transformation $f \in \operatorname{Möb}\left(\hat{\mathbb{R}}^{n}\right)$ is said to be pure parabolic if it is conjugate to a translation $\boldsymbol{x} \mapsto \boldsymbol{x}+\boldsymbol{u}$, and screw parabolic otherwise.

We now observe the case of $n=3$. Since every $P \in S O(3)$ has an eigenvalue of 1 , we have the following:

Lemma 2.5. Let $f \in \operatorname{Möb}\left(\hat{\mathbb{R}}^{3}\right)$.

(1) If $f$ is parabolic, it is conjugate in $\mathrm{Möb}\left(\hat{\mathbb{R}}^{3}\right)$ to a transformation given by

$$
\left(\begin{array}{l}
x_{1} \\
x_{2} \\
x_{3}
\end{array}\right) \mapsto\left(\begin{array}{ccc}
\cos \theta & -\sin \theta & 0 \\
\sin \theta & \cos \theta & 0 \\
0 & 0 & 1
\end{array}\right)\left(\begin{array}{l}
x_{1} \\
x_{2} \\
x_{3}
\end{array}\right)+\left(\begin{array}{l}
0 \\
0 \\
1
\end{array}\right),
$$

where $0 \leq \theta<2 \pi$. (Then $f$ is pure parabolic if and only if $\theta=0$.)

(2) If $f$ is loxodromic, it is conjugate in $\operatorname{Möb}\left(\hat{\mathbb{R}}^{3}\right)$ to a transformation given by

$$
\left(\begin{array}{l}
x_{1} \\
x_{2} \\
x_{3}
\end{array}\right) \mapsto \lambda\left(\begin{array}{ccc}
\cos \theta & -\sin \theta & 0 \\
\sin \theta & \cos \theta & 0 \\
0 & 0 & 1
\end{array}\right)\left(\begin{array}{l}
x_{1} \\
x_{2} \\
x_{3}
\end{array}\right),
$$

where $\lambda>1$ and $0 \leq \theta<2 \pi$.

It is worth noting that in $\operatorname{Möb}\left(\hat{\mathbb{R}}^{3}\right)$ a pure parabolic transformation is topologically conjugate to any screw parabolic transformation. More precisely, a translation 
$\left(x_{1}, x_{2}, x_{3}\right) \mapsto\left(x_{1}, x_{2}, x_{3}\right)+(0,0,1)$ is conjugate to a screw parabolic transformation of the form $(2.3)$ by an orientation-preserving homeomorphism $f: \hat{\mathbb{R}}^{3} \rightarrow \hat{\mathbb{R}}^{3}$ defined by

$$
\left(\begin{array}{l}
x_{1} \\
x_{2} \\
x_{3}
\end{array}\right) \mapsto\left(\begin{array}{ccc}
\cos \left(x_{3} \theta\right) & -\sin \left(x_{3} \theta\right) & 0 \\
\sin \left(x_{3} \theta\right) & \cos \left(x_{3} \theta\right) & 0 \\
0 & 0 & 1
\end{array}\right)\left(\begin{array}{l}
x_{1} \\
x_{2} \\
x_{3}
\end{array}\right)
$$

2.6. Kleinian groups. A discrete subgroup $\Gamma$ of $\operatorname{Isom}\left(\mathbf{H}^{n+1}\right)=\operatorname{Möb}\left(\mathbb{R}^{n}\right)$ is called an $(n+1)$-dimensional Kleinian group. It is known that $\Gamma$ is a Kleinian group if and only if $\Gamma$ acts properly discontinuously on $\mathbf{H}^{n+1}$; that is, given any compact subset $K \subset \mathbf{H}^{n+1}$, the set $\{\gamma \in \Gamma: \gamma(K) \cap K \neq \emptyset\}$ is finite.

Let $\Gamma$ be an $(n+1)$-dimensional Kleinian group. The domain of discontinuity $\Omega(\Gamma) \subset \hat{\mathbb{R}}^{n}$ of $\Gamma$ is defined to be the set of points $\boldsymbol{x} \in \hat{\mathbb{R}}^{n}$ such that there is a neighborhood $U$ of $\boldsymbol{x}$ such that the set $\{\gamma \in \Gamma: \gamma(U) \cap U=\emptyset\}$ is finite. The complement of $\Omega(\Gamma)$ in $\hat{\mathbb{R}}^{n}$ is called the limit set of $\Gamma$, and denoted by $\Lambda(\Gamma)$.

2.7. Notation. Throughout this paper, we use the following notation:

- If $S$ is a subset of a group $G$, we denote by $\langle S\rangle \subset G$ the subgroup generated by $S$.

- According to the usual convention, a Möbius transformation $f \in \operatorname{Möb}\left(\hat{\mathbb{R}}^{2}\right)$ is written in a linear fractional form

$$
f(\tau)=\frac{a \tau+b}{c \tau+d} \quad(a, b, c, d \in \mathbb{C})
$$

by identifying $\hat{\mathbb{R}}^{2}$ with the Riemann sphere $\hat{\mathbb{C}}$.

- A Möbius transformation $f: \hat{\mathbb{R}}^{3} \rightarrow \hat{\mathbb{R}}^{3}$ in $\operatorname{Möb}\left(\hat{\mathbb{R}}^{3}\right)$ is written as a function $f(\boldsymbol{x})$ of $\boldsymbol{x}=(x, y, z) \in \mathbb{R}^{3}$.

- We denote by $P_{z=0}$ the plane $\left\{(x, y, z) \in \mathbb{R}^{3}: z=0\right\}$ and by $P_{r=0}$ the plane $\left\{(p, q, r) \in \mathbb{R}^{3}: r=0\right\}$ and so on. In addition, we write $\hat{P}_{z=0}=P_{z=0} \cup\{\infty\}$ and $\hat{P}_{r=0}=P_{r=0} \cup\{\infty\}$ and so on.

\section{Groups in Möb $\left(\hat{\mathbb{R}}^{2}\right)$}

In this section, we recall some basic facts of Kleinian groups in $\operatorname{Möb}\left(\hat{\mathbb{R}}^{2}\right)$ which are isomorphic to the fundamental groups of a thrice-punctured sphere, a fourthpunctured sphere or a punctured torus. We refer the reader to Kra [9] and KeenSeries [10].

A surface $\Sigma_{g, n}$ of type $(g, n)$ is an oriented closed surface of genus $g$ with $n$ punctures. If $n>0$, the fundamental group $\pi_{1}\left(\Sigma_{g, n}\right)$ of $\Sigma_{g, n}$ is a free group. A representation $\rho: \pi_{1}\left(\Sigma_{g, n}\right) \rightarrow \operatorname{Möb}\left(\hat{\mathbb{R}}^{2}\right)$ is said to be type-preserving if it takes a loop surrounding a puncture to a parabolic transformation. In this section, we consider images of faithful type-preserving representations $\rho: \pi_{1}\left(\Sigma_{g, n}\right) \rightarrow \operatorname{Möb}\left(\hat{\mathbb{R}}^{2}\right)$ for $(g, n)=(0,3),(0,4)$ and $(1,1)$. We choose generators for $\pi_{1}\left(\Sigma_{0,3}\right)=\langle b, c\rangle$, $\pi_{1}\left(\Sigma_{0,4}\right)=\langle b, c, d\rangle$ and $\pi_{1}\left(\Sigma_{1,1}\right)=\langle a, b\rangle$ as in Figure 3. We define groups of type $(0,3),(0,4)$ and $(1,1)$ in $\operatorname{Möb}\left(\hat{\mathbb{R}}^{2}\right)$ as follows:

Definition 3.1. Let $\Gamma$ be a subgroup of $\operatorname{Möb}\left(\hat{\mathbb{R}}^{2}\right)$.

- $\Gamma$ is said to be of type $(0,3)$ if it is the image of a faithful, type-preserving representation $\rho: \pi_{1}\left(\Sigma_{0,3}\right)=\langle b, c\rangle \rightarrow \operatorname{Möb}\left(\hat{\mathbb{R}}^{2}\right)$, or equivalently, if it is a rank-2 free group $\langle\beta, \gamma\rangle$ such that $\beta, \gamma$ and $\beta \gamma$ are parabolic. 

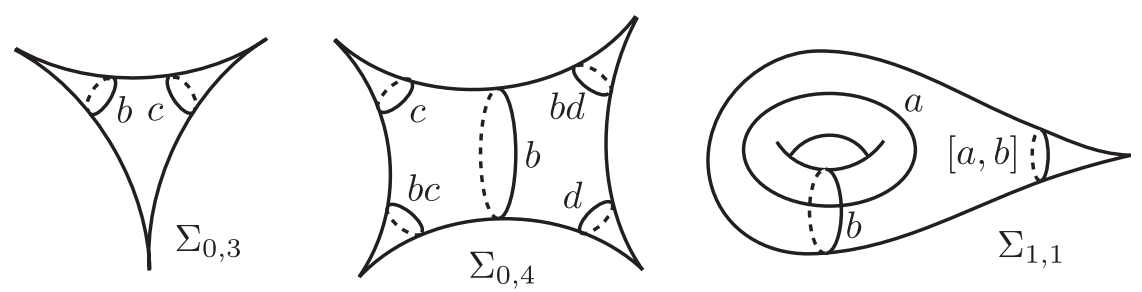

FiguRE 3. Surfaces $\Sigma_{0,3}, \Sigma_{0,4}$ and $\Sigma_{1,1}$, and free homotopy classes of elements of their fundamental groups.

- $\Gamma$ is said to be of type $(0,4)$ if it is the image of a faithful, type-preserving representation $\rho: \pi_{1}\left(\Sigma_{0,4}\right)=\langle b, c, d\rangle \rightarrow \operatorname{Möb}\left(\hat{\mathbb{R}}^{2}\right)$ such that $\rho(b)$ is parabolic, or equivalently, if it is a rank-3 free group $\langle\beta, \gamma, \delta\rangle$ such that $\beta, \gamma, \delta, \beta \gamma$ and $\beta \delta$ are parabolic.

- $\Gamma$ is said to be of type $(1,1)$ if it is the image of a faithful, type-preserving representation $\rho: \pi_{1}\left(\Sigma_{1,1}\right)=\langle a, b\rangle \rightarrow \operatorname{Möb}\left(\hat{\mathbb{R}}^{2}\right)$ such that $\rho(b)$ is parabolic, or equivalently, if it is a rank-2 free group $\langle\alpha, \beta\rangle$ such that $\beta$ and $[\alpha, \beta]$ is parabolic.

3.1. Groups of type $(0,3)$. Observe that the subgroup $\langle B, C\rangle$ of $\operatorname{Möb}\left(\hat{\mathbb{R}}^{2}\right)$ generated by

$$
B(\tau)=\tau+2 \quad \text { and } \quad C(\tau)=\frac{1}{B\left(\frac{1}{\tau}\right)}=\frac{\tau}{2 \tau+1}
$$

is of type $(0,3)$. It is known that any group of type $(0,3)$ in $\operatorname{Möb}\left(\hat{\mathbb{R}}^{2}\right)$ is conjugate to this group:

Lemma 3.2. Let $\Gamma=\langle\beta, \gamma\rangle$ be a rank-2 free subgroup of $\operatorname{Möb}\left(\hat{\mathbb{R}}^{2}\right)$ such that $\beta, \gamma$ and $\beta \gamma$ are parabolic. Then $\Gamma$ is conjugate in $\operatorname{Möb}\left(\hat{\mathbb{R}}^{2}\right)$ to the group $\langle B, C\rangle$ defined above.

3.2. Groups of type $(0,4)$. For a given $\mu \in \mathbb{C}$, let

$$
H_{\mu}=\left\langle B, C, D_{\mu}\right\rangle
$$

be the group in $\operatorname{Möb}\left(\hat{\mathbb{R}}^{2}\right)$ generated by

$$
B(\tau)=\tau+2, \quad C(\tau)=\frac{\tau}{2 \tau+1} \quad \text { and } \quad D_{\mu}(\tau)=C(\tau-\mu)+\mu .
$$

It is known that any group of type $(0,4)$ in $\operatorname{Möb}\left(\hat{\mathbb{R}}^{2}\right)$ is normalized in this form:

Lemma 3.3. Let $\Gamma=\langle\beta, \gamma, \delta\rangle$ be a rank-3 free subgroup of $\mathrm{Möb}\left(\hat{\mathbb{R}}^{2}\right)$ such that $\beta, \gamma, \delta, \beta \gamma$ and $\beta \delta$ are parabolic. Then $\Gamma$ is conjugate in $\operatorname{Möb}\left(\hat{\mathbb{R}}^{2}\right)$ to $H_{\mu}=\left\langle B, C, D_{\mu}\right\rangle$ for some $\mu \in \mathbb{C}$.

A fundamental domain for $H_{\mu}$ with $\operatorname{Im} \mu \geq 1$ is given in the left of Figure 4. The Maskit slice of groups of type $(0,4)$ in $\operatorname{Möb}\left(\hat{\mathbb{R}}^{2}\right)$ is defined by

$$
\mathcal{M}_{0,4}=\left\{\mu \in \mathbb{C}: H_{\mu}=\left\langle B, C, D_{\mu}\right\rangle \text { is a rank-3 free Kleinian group }\right\} .
$$

Some basic properties of $\mathcal{M}_{0,4}$ can be found in the next subsection. 

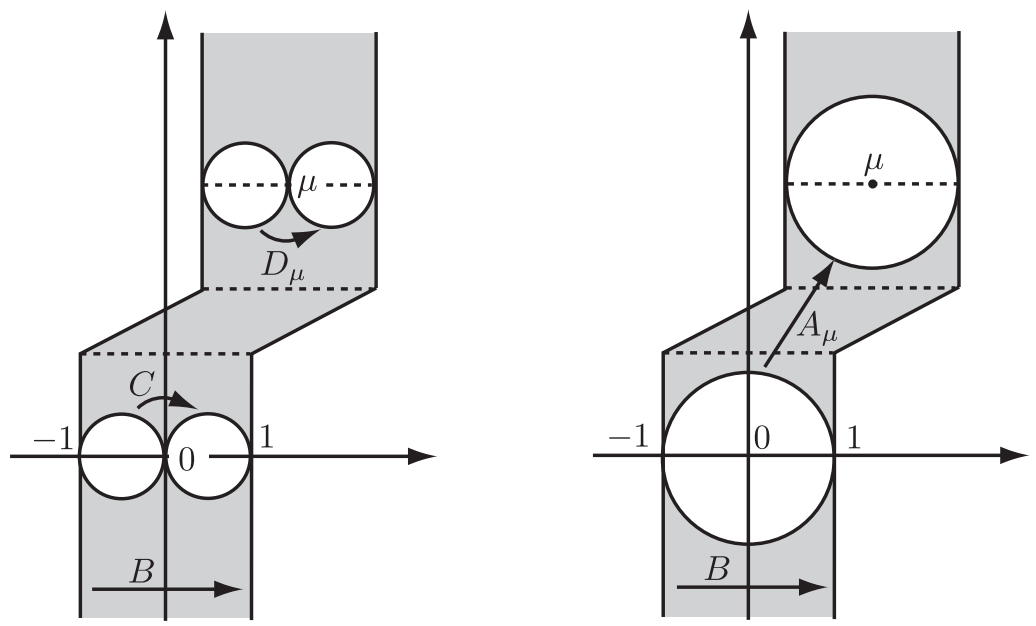

Figure 4. Fundamental domains (the shaded regions) for $H_{\mu}$ (left) and $G_{\mu}$ (right).

3.3. Groups of type $(1,1)$. For a given $\mu \in \mathbb{C}$, let

$$
G_{\mu}=\left\langle A_{\mu}, B\right\rangle
$$

be the group in $\operatorname{Möb}\left(\hat{\mathbb{R}}^{2}\right)$ generated by

$$
A_{\mu}(\tau)=\frac{1}{\tau}+\mu \quad \text { and } \quad B(\tau)=\tau+2 .
$$

It is known that any group of type $(1,1)$ in $\operatorname{Möb}\left(\hat{\mathbb{R}}^{2}\right)$ is normalized in this form:

Lemma 3.4. Let $\Gamma=\langle\alpha, \beta\rangle$ be a rank-2 free subgroup of $\operatorname{Möb}\left(\hat{\mathbb{R}}^{2}\right)$ such that $\beta$ and $[\alpha, \beta]$ are parabolic. Then $\Gamma$ is conjugate in $\operatorname{Möb}\left(\hat{\mathbb{R}}^{2}\right)$ to $G_{\mu}=\left\langle A_{\mu}, B\right\rangle$ for some $\mu \in \mathbb{C}$.

A fundamental domain for $G_{\mu}$ with $\operatorname{Im} \mu \geq 2$ is given in the right of Figure 4 . The Maskit slice of groups of type $(1,1)$ in $\operatorname{Möb}\left(\hat{\mathbb{R}}^{2}\right)$ is defined by

$$
\mathcal{M}_{1,1}=\left\{\mu \in \mathbb{C}: G_{\mu}=\left\langle A_{\mu}, B\right\rangle \text { is a rank-2 free Kleinian group }\right\}
$$

(see also Figure 5). It is easy to see that $\mathcal{M}_{1,1}$ is invariant under the maps $\mu \mapsto \mu+2$ and $\mu \mapsto-\mu$, and that $\mathcal{M}_{1,1}$ contains the set $\{\mu \in \mathbb{C}:|\operatorname{Im} \mu| \geq 2\}$. It is also known that the Maskit slice $\mathcal{M}_{1,1}$ is contained in the set $\{\mu \in \mathbb{C}:|\operatorname{Im} \mu|>1\}$ (cf. [10]). Furthermore, Minsky [14] showed that $\mathcal{M}_{1,1}$ consists of two connected components and that each connected component of $\mathcal{M}_{1,1}$ plus $\{\infty\}$ is a closed topological disc in $\hat{\mathbb{C}}$.

We now mention the relationship between the Maskit slices $\mathcal{M}_{0,4}$ and $\mathcal{M}_{1,1}$. First observe for every $\mu \in \mathbb{C}$ that

$$
C=A_{\mu}^{-1} B A_{\mu} \quad \text { and } \quad D_{\mu}=A_{\mu} B A_{\mu}^{-1} .
$$

Thus $H_{\mu}=\left\langle B, C, D_{\mu}\right\rangle$ is always a subgroup of $G_{\mu}=\left\langle A_{\mu}, B\right\rangle$. This implies that $\mathcal{M}_{1,1} \subset \mathcal{M}_{0,4}$. Furthermore, it is known by Kra that $\mathcal{M}_{0,4}$ is similar to $\mathcal{M}_{1,1}$ :

Theorem 3.5 (Kra [9]). The map $\tau \mapsto 2 \tau$ of $\mathbb{C}$ onto itself induces a bijective map from $\mathcal{M}_{0,4}$ onto $\mathcal{M}_{1,1}$. 


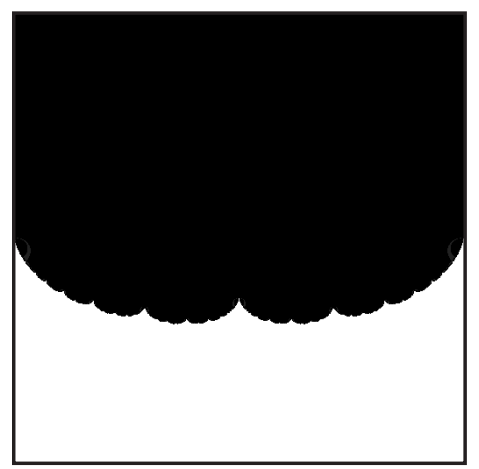

Figure 5. The Maskit slice $\mathcal{M}_{1,1}$ (black part) restricted to the domain $\{\mu \in \mathbb{C}: 0 \leq \operatorname{Re} \mu \leq 2,1 \leq \operatorname{Im} \mu \leq 3\}$.

\section{Groups IN Möb $\left(\hat{\mathbb{R}}^{3}\right)$}

We define 4-dimensional analogues of 3-dimensional groups of types $(0,3),(0,4)$ and $(1,1)$ as follows:

Definition 4.1. Let $\Gamma$ be a subgroup of $\operatorname{Möb}\left(\hat{\mathbb{R}}^{3}\right)$.

- $\Gamma$ is said to be of type $(0,3)$ if it is a rank-2 free group $\langle\beta, \gamma\rangle$ such that $\beta, \gamma$ and $\beta \gamma$ are pure parabolic.

- $\Gamma$ is said to be of type $(0,4)$ if it is a rank-3 free group $\langle\beta, \gamma, \delta\rangle$ such that $\beta, \gamma, \delta, \beta \gamma$ and $\beta \delta$ are pure parabolic.

- $\Gamma$ is said to be of type $(1,1)$ if it is a rank-2 free group $\langle\alpha, \beta\rangle$ such that $\beta$ and $[\alpha, \beta]$ are pure parabolic.

As mentioned in Section 1, Introduction, it is just a technical reason why we assume some elements of these groups are (not only parabolic but also) pure parabolic. In this section, we study normalizations of these groups in $\operatorname{Möb}\left(\hat{\mathbb{R}}^{3}\right)$ up to conjugation.

4.1. Groups of type $(0,3)$ in $\operatorname{Möb}\left(\hat{\mathbb{R}}^{3}\right)$. Observe that the Poincaré extension of the group $\langle B(\tau)=\tau+2, C(\tau)=\tau /(2 \tau+1)\rangle \subset \operatorname{Möb}\left(\hat{\mathbb{R}}^{2}\right)$ acting on $\hat{\mathbb{C}}$ is given by

$$
\langle B(\boldsymbol{x})=\boldsymbol{x}+(2,0,0), C(\boldsymbol{x})=J B J(\boldsymbol{x})\rangle \subset \operatorname{Möb}\left(\hat{\mathbb{R}}^{3}\right),
$$

where $\hat{\mathbb{C}}$ is identified with $\hat{P}_{z=0} \subset \hat{\mathbb{R}}^{3}$ via the map $\tau=x+i y \mapsto \boldsymbol{x}=(x, y, 0)$, and $J(\boldsymbol{x})=\boldsymbol{x} /|\boldsymbol{x}|^{2}$ is the inversion in the unit sphere. (Here and hereafter, we use the same symbols $B, C$ for the Poincaré extensions of $B, C \in \operatorname{Möb}\left(\hat{\mathbb{R}}^{2}\right)$ by abuse of notation.) Any group of type $(0,3)$ in $\operatorname{Möb}\left(\hat{\mathbb{R}}^{3}\right)$ is conjugate to this group:

Theorem 4.2. Let $\Gamma=\langle\beta, \gamma\rangle$ be a rank-2 free subgroup of $\mathrm{Möb}\left(\hat{\mathbb{R}}^{3}\right)$ such that $\beta$, $\gamma$ and $\beta \gamma$ are pure parabolic. Then $\Gamma$ is conjugate in $\operatorname{Möb}\left(\hat{\mathbb{R}}^{3}\right)$ to the group $\langle B, C\rangle$ defined above.

Proof. Observe first that $\operatorname{Fix}(\beta) \neq \operatorname{Fix}(\gamma)$. In fact, if $\operatorname{Fix}(\beta)=\operatorname{Fix}(\gamma), \beta$ commutes with $\gamma$ since both $\beta, \gamma$ are pure parabolic. This contradicts that $\langle\beta, \gamma\rangle$ is free. Therefore, after conjugating the group $\langle\beta, \gamma\rangle$ in $\operatorname{Möb}\left(\hat{\mathbb{R}}^{3}\right)$ if necessary, we may assume that $\beta(\infty)=\infty$ and $\gamma(\mathbf{0})=\mathbf{0}$. Observe that there exist lines $l$ and $m$ through the 
origin $\mathbf{0}$ which are invariant under the actions of $\langle\beta\rangle$ and $\langle\gamma\rangle$, respectively. After conjugating by an orthonormal translation if necessary, we may assume that both $l, m$ lie in the sphere $\hat{P}_{z=0}$. Then one can see that the group $\langle\beta, \gamma\rangle$ preserves the sphere $\hat{P}_{z=0}$ and its orientation. Thus the result follows from Lemma 3.2.

Remark. Recently, Y. Kim and K. Sakugawa independently announced (oral communication) that there is a continuous family of rank-2 free Kleinian groups $\Gamma=$ $\langle\beta, \gamma\rangle$ in $\operatorname{Möb}\left(\hat{\mathbb{R}}^{3}\right)$ such that $\beta, \gamma$ and $\beta \gamma$ are (screw) parabolic.

In the next subsection, we need the following:

Corollary 4.3. Let $\langle\beta, \gamma\rangle$ be a rank-2 free subgroup of $\operatorname{Möb}\left(\hat{\mathbb{R}}^{3}\right)$ such that $\beta, \gamma$ and $\beta \gamma$ are pure parabolic. If $\beta=B$ and $\gamma(\mathbf{0})=\mathbf{0}$, then $\gamma=C^{ \pm 1}$; that is, $\langle\beta, \gamma\rangle=\langle B, C\rangle$.

Proof. By Theorem 4.2, there is a transformation $f \in \operatorname{Möb}\left(\hat{\mathbb{R}}^{3}\right)$ which conjugates $\langle B, \gamma\rangle$ to $\langle B, C\rangle$. Since $J$ conjugates $B$ and $C$ each other, we may assume that $f B f^{-1}=B$ and $f \gamma f^{-1}=C^{ \pm 1}$. This implies that $f(\mathbf{0})=\mathbf{0}$ and $f(\infty)=\infty$. Then by Lemma 2.1, we have $f=\lambda P$, where $\lambda>0$ and $P \in S O(3)$. Moreover, it follows from the condition $f B f^{-1}=B$ that $f$ is a rotation along the $x$-axis. Since such $f$ conjugates $C$ to itself, we have $\gamma=f^{-1} C^{ \pm 1} f=C^{ \pm 1}$.

4.2. Groups of type $(0,4)$. In this subsection, we will show that every group of type $(0,4)$ in $\operatorname{Möb}\left(\hat{\mathbb{R}}^{3}\right)$ is conjugate to a group of type $(0,4)$ in $\operatorname{Möb}\left(\hat{\mathbb{R}}^{2}\right)$.

For a given $\boldsymbol{p}=(p, q, r) \in \mathbb{R}^{3}$, let

$$
H_{\boldsymbol{p}}=\left\langle B, C, D_{\boldsymbol{p}}\right\rangle
$$

be the subgroup of Möb $\left(\hat{\mathbb{R}}^{3}\right)$ generated by

$$
B(\boldsymbol{x})=\boldsymbol{x}+(2,0,0), \quad C(\boldsymbol{x})=J B J(\boldsymbol{x}) \quad \text { and } \quad D_{\boldsymbol{p}}(\boldsymbol{x})=C(\boldsymbol{x}-\boldsymbol{p})+\boldsymbol{p} .
$$

Observe that if $\boldsymbol{p}$ lies in the plane $P_{r=0}$, the group $H_{\boldsymbol{p}}$ for $\boldsymbol{p}=(p, q, 0)$ is the Poincaré extension of the group $H_{\mu}=\left\langle B, C, D_{\mu}\right\rangle$ for $\mu=p+i q$ defined in 3.2.

Theorem 4.4. Let $\Gamma=\langle\beta, \gamma, \delta\rangle$ be a rank-3 free subgroup of $\mathrm{Möb}\left(\hat{\mathbb{R}}^{3}\right)$ such that $\beta, \gamma, \delta, \beta \gamma$ and $\beta \delta$ are pure parabolic. Then $\Gamma$ is conjugate in $\operatorname{Möb}\left(\hat{\mathbb{R}}^{3}\right)$ to $H_{p}=$ $\left\langle B, C, D_{\boldsymbol{p}}\right\rangle$ for some $\boldsymbol{p}=(p, q, 0) \in P_{r=0}$.

Proof. Note that the subgroups $\langle\beta, \gamma\rangle,\langle\beta, \delta\rangle$ of $\langle\beta, \gamma, \delta\rangle$ are of type $(0,3)$ in $\operatorname{Möb}\left(\hat{\mathbb{R}}^{3}\right)$. Therefore, after conjugating $\langle\beta, \gamma, \delta\rangle$ in $\operatorname{Möb}\left(\hat{\mathbb{R}}^{3}\right)$ if necessary, we may assume that $\beta=B$ and $\operatorname{Fix}(\gamma)=\mathbf{0}$, and hence that $\langle\beta, \gamma\rangle=\langle B, C\rangle$ by Corollary 4.3. Since a rotation along the $x$-axis conjugates the group $\langle B, C\rangle$ to itself, we may also assume that the fixed point $\boldsymbol{p}:=\operatorname{Fix}(\delta)$ of $\delta$ lies in the plane $P_{z=0}$. To obtain the result, it suffices to show that $\langle\beta, \delta\rangle=\left\langle B, D_{\boldsymbol{p}}\right\rangle$. Now let us conjugate the group $\langle B, \delta\rangle$ by the translation $T_{\boldsymbol{p}}^{-1}(\boldsymbol{x})=\boldsymbol{x}-\boldsymbol{p}$ to the group $T_{\boldsymbol{p}}^{-1}\langle B, \delta\rangle T_{\boldsymbol{p}}=\left\langle B, T_{\boldsymbol{p}}^{-1} \delta T_{\boldsymbol{p}}\right\rangle$. Since $T_{\boldsymbol{p}}^{-1} \delta T_{\boldsymbol{p}}$ fixes $\mathbf{0}$, we have $\left\langle B, T_{\boldsymbol{p}}^{-1} \delta T_{\boldsymbol{p}}\right\rangle=\langle B, C\rangle$ from Corollary 4.3. Thus we conclude that $\langle B, \delta\rangle=T_{\boldsymbol{p}}\langle B, C\rangle T_{\boldsymbol{p}}^{-1}=\left\langle B, D_{\boldsymbol{p}}\right\rangle$.

4.3. Groups of type $(1,1)$. In this subsection, we will obtain a normalization of groups of type $(1,1)$ in $\operatorname{Möb}\left(\hat{\mathbb{R}}^{3}\right)$. In contrast to the case of groups of type $(0,4)$, we will see that the space of groups of type $(1,1)$ in $\operatorname{Möb}\left(\hat{\mathbb{R}}^{3}\right)$ is strictly larger than the space of groups of type $(1,1)$ in $\operatorname{Möb}\left(\hat{\mathbb{R}}^{2}\right)$. 
For a given $\boldsymbol{p} \in \mathbb{R}^{3}$, let

$$
G_{p}=\left\langle A_{p}, B\right\rangle
$$

be the subgroup of $\operatorname{Möb}\left(\hat{\mathbb{R}}^{3}\right)$ generated by

$$
A_{\boldsymbol{p}}(\boldsymbol{x})=\hat{J} J(\boldsymbol{x})+\boldsymbol{p} \quad \text { and } \quad B(\boldsymbol{x})=\boldsymbol{x}+(2,0,0),
$$

where

$$
\hat{J}(x, y, z)=(x,-y, z)
$$

is the inversion in the sphere $\hat{P}_{y=0}$. Observe for every $\boldsymbol{p} \in \mathbb{R}^{3}$ that

$$
C=A_{p}^{-1} B A_{p} \quad \text { and } \quad D_{p}=A_{p} B A_{p}^{-1} .
$$

Thus $H_{\boldsymbol{p}}=\left\langle B, C, D_{\boldsymbol{p}}\right\rangle$ is always a subgroup of $G_{\boldsymbol{p}}=\left\langle A_{\boldsymbol{p}}, B\right\rangle$. In addition, observe that if $\boldsymbol{p}$ lies in the plane $P_{r=0}$, the group $G_{\boldsymbol{p}}$ for $\boldsymbol{p}=(p, q, 0)$ is the Poincaré extension of the group $G_{\mu}=\left\langle A_{\mu}, B\right\rangle$ for $\mu=p+i q$ defined in 3.3. The next theorem reveals that every group of type $(1,1)$ in $\operatorname{Möb}\left(\hat{\mathbb{R}}^{3}\right)$ is conjugate to the group $G_{\boldsymbol{p}}$ for some $\boldsymbol{p} \in \mathbb{R}^{3}$. (We will prove a slightly stronger statement.)

Theorem 4.5. Let $\Gamma=\langle\alpha, \beta\rangle$ be a rank-2 free group in $\operatorname{Möb}\left(\hat{\mathbb{R}}^{3}\right)$ such that $\beta$ is pure parabolic and $[\alpha, \beta]$ is (not necessarily pure) parabolic. Then $\Gamma$ is conjugate in $\operatorname{Möb}\left(\hat{\mathbb{R}}^{3}\right)$ to $G_{\boldsymbol{p}}=\left\langle A_{\boldsymbol{p}}, B\right\rangle$ for some $\boldsymbol{p}=(p, q, r) \in \mathbb{R}^{3}$.

Proof. We first show that $\operatorname{Fix}(\alpha) \cap \operatorname{Fix}(\beta)=\emptyset$. If not, we may assume that $\infty \in$ $\operatorname{Fix}(\alpha) \cap \operatorname{Fix}(\beta)$. It then follows from Lemma 2.1 that $\alpha(\boldsymbol{x})=\lambda P(\boldsymbol{x})+\boldsymbol{u}$ and $\beta(\boldsymbol{x})=\boldsymbol{x}+\boldsymbol{v}$ for some $\lambda>0, P \in S O(3)$ and $\boldsymbol{u}, \boldsymbol{v} \in \mathbb{R}^{3}$. Then a calculation yields $[\alpha, \beta](\boldsymbol{x})=\boldsymbol{x}+\lambda P(\boldsymbol{v})-\boldsymbol{v}$. Thus $[\alpha, \beta]$ is pure parabolic with the fixed point $\infty$. This implies that $\beta$ commutes with $[\alpha, \beta]$, which contradicts that $\langle\alpha, \beta\rangle$ is free.

Therefore we may assume that $\beta(\infty)=\infty$ and $\alpha(\mathbf{0})=\infty$. It then follows from Lemma 2.1 that $\alpha, \beta$ are of the forms $\alpha(\boldsymbol{x})=\lambda P J(\boldsymbol{x})+\boldsymbol{p}$ and $\beta(\boldsymbol{x})=\boldsymbol{x}+\boldsymbol{u}$ for some $\lambda>0, P \in O(3) \backslash S O(3)$ and $\boldsymbol{p}, \boldsymbol{u} \in \mathbb{R}^{3}$. We may further conjugate the group $\langle\alpha, \beta\rangle$ by a transformation $f \in \operatorname{Möb}\left(\hat{\mathbb{R}}^{3}\right)$ fixing $\mathbf{0}$ and $\infty$ without changing our assumptions $\beta(\infty)=\infty, \alpha(\mathbf{0})=\infty$. Such a transformation is of the form $f=\lambda^{\prime} Q \in \operatorname{Möb}\left(\hat{\mathbb{R}}^{3}\right)$ with $\lambda^{\prime}>0, Q \in S O(3)$ by Lemma 2.3. Therefore, after conjugating $\langle\alpha, \beta\rangle$ by a magnification if necessary, we may assume that the radius of the isometric sphere of $\alpha$ equals 1 , and hence that $\alpha, \beta$ are of the forms $\alpha(\boldsymbol{x})=P J(\boldsymbol{x})+\boldsymbol{p}$ and $\beta(\boldsymbol{x})=\boldsymbol{x}+\boldsymbol{u}$. In addition, we claim that we may also assume that

$$
\boldsymbol{u}=(u, v, 0) \quad \text { and } \quad P^{-1}(\boldsymbol{u})=(u,-v, 0)
$$

for some $u, v \geq 0$ after conjugating $\langle\alpha, \beta\rangle$ by a suitable orthonormal transformation if necessary. In fact, choose $Q \in S O(3)$ so that $Q(\boldsymbol{u})=(u, v, 0)$ and $Q P^{-1}(\boldsymbol{u})=$ $(u,-v, 0)$. Then $Q$ conjugates $\alpha(\boldsymbol{x})=P J(\boldsymbol{x})+\boldsymbol{p}$ to $Q \alpha Q^{-1}(\boldsymbol{x})=Q P Q^{-1} J(\boldsymbol{x})+$ $Q(\boldsymbol{p})$ and $\beta(\boldsymbol{x})=\boldsymbol{x}+\boldsymbol{u}$ to $Q \beta Q^{-1}(\boldsymbol{x})=\boldsymbol{x}+Q(\boldsymbol{u})$. Now observe that $\left(Q P Q^{-1}\right)^{-1}$ takes $Q(\boldsymbol{u})=(u, v, 0)$ to $Q P^{-1}(\boldsymbol{u})=(u,-v, 0)$. Therefore, replacing $Q P Q^{-1}, Q(\boldsymbol{p})$ and $Q(\boldsymbol{u})$ by $P, \boldsymbol{p}$ and $\boldsymbol{u}$, respectively, we obtain the claim.

Next we will show that $\boldsymbol{u}=P^{-1}(\boldsymbol{u})=(2,0,0)$ by using the condition that $[\alpha, \beta]$ is parabolic. Recall that $T_{\boldsymbol{v}}$ denotes the translation $\boldsymbol{x} \mapsto \boldsymbol{x}+\boldsymbol{v}$ with $\boldsymbol{v} \in \mathbb{R}^{3}$. Using this notation, we have $\alpha(\boldsymbol{x})=T_{\boldsymbol{p}} P J(\boldsymbol{x}), \beta(\boldsymbol{x})=T_{\boldsymbol{u}}(\boldsymbol{x}), \alpha^{-1}=J P^{-1} T_{-\boldsymbol{p}}(\boldsymbol{x})$ and $\beta^{-1}(\boldsymbol{x})=T_{-\boldsymbol{u}}(\boldsymbol{x})$. Then the transformation $(\beta \alpha)^{-1}[\alpha, \beta] \beta \alpha=\alpha^{-1} \beta^{-1} \alpha \beta$ is 
calculated as

$$
\begin{aligned}
\alpha^{-1} \beta^{-1} \alpha \beta(\boldsymbol{x}) & =J P^{-1} T_{-\boldsymbol{p}} T_{-\boldsymbol{u}} T_{\boldsymbol{p}} P J T_{\boldsymbol{u}}(\boldsymbol{x}) \\
& =J P^{-1} T_{-\boldsymbol{u}} P J T_{\boldsymbol{u}}(\boldsymbol{x}) \\
& =J T_{-P^{-1}(\boldsymbol{u})} J T_{\boldsymbol{u}}(\boldsymbol{x}),
\end{aligned}
$$

where the last equality follows from $P^{-1} T_{-\boldsymbol{u}} P(\boldsymbol{x})=P^{-1}(P(\boldsymbol{x})-\boldsymbol{u})=\boldsymbol{x}-P^{-1}(\boldsymbol{u})=$ $T_{-P^{-1}(\boldsymbol{u})}(\boldsymbol{x})$. Since $\boldsymbol{u}=(u, v, 0)$ and $P^{-1}(\boldsymbol{u})=(u,-v, 0)$ lie in the plane $P_{z=0}$, the transformation $\alpha^{-1} \beta^{-1} \alpha \beta=J T_{-P^{-1}(\boldsymbol{u})} J T_{\boldsymbol{u}}$ preserves the sphere $\hat{P}_{z=0}$ and its orientation. By letting $\mu=u+i v$ and identifying $\hat{P}_{z=0}$ with $\hat{\mathbb{C}}$ via the map $\boldsymbol{x}=(x, y, 0) \mapsto \tau=x+i y$, we see that the action of $\alpha^{-1} \beta^{-1} \alpha \beta$ restricted to the sphere $\hat{P}_{z=0} \cong \hat{\mathbb{C}}$ is a Möbius transformation

$$
\tau \mapsto \frac{\tau+\mu}{-\mu \tau+1-\mu^{2}},
$$

whose matrix representation is

$$
\left(\begin{array}{cc}
1 & \mu \\
-\mu & 1-\mu^{2}
\end{array}\right) \in \mathrm{PSL}_{2}(\mathbb{C})
$$

Since $\alpha^{-1} \beta^{-1} \alpha \beta$ is parabolic and $\operatorname{Re} \mu=u \geq 0$, we conclude that $\mu=2$, and hence that $\boldsymbol{u}=P^{-1}(\boldsymbol{u})=(2,0,0)$.

Now let us denote by $R_{\theta} \in S O(3)$ the rotation of angle $\theta \in \mathbb{R}$ along the $x$-axis. Since $P \in O(3) \backslash S O(3)$ and $P(\boldsymbol{u})=\boldsymbol{u}$, we have $P=R_{\varphi} \hat{J}$ for some $\varphi \in \mathbb{R}$. Noting that $\hat{J} R_{-\theta}=R_{\theta} \hat{J}$ and $J R_{\theta}=R_{\theta} J$ for every $\theta \in \mathbb{R}$, we obtain

$$
\begin{aligned}
& R_{\theta} \alpha R_{\theta}^{-1}(\boldsymbol{x})=R_{\theta}\left(R_{\varphi} \hat{J} J R_{-\theta}(\boldsymbol{x})+\boldsymbol{p}\right)=R_{\varphi+2 \theta} \hat{J} J(\boldsymbol{x})+R_{\theta}(\boldsymbol{p}), \\
& R_{\theta} \beta R_{\theta}^{-1}(\boldsymbol{x})=\beta(\boldsymbol{x}) .
\end{aligned}
$$

Therefore, after conjugating $\langle\alpha, \beta\rangle$ by $R_{\theta}$ with $\theta \equiv-\varphi / 2(\bmod \pi)$ if necessary, we may assume that $\alpha, \beta$ are of the forms $\alpha(\boldsymbol{x})=A_{\boldsymbol{p}}(\boldsymbol{x})=\hat{J} J(\boldsymbol{x})+\boldsymbol{p}$ and $\beta(\boldsymbol{x})=$ $B(\boldsymbol{x})=\boldsymbol{x}+(2,0,0)$. Thus we obtain the desired normalization.

Remark. The condition that the group $\Gamma=\langle\alpha, \beta\rangle$ is free is only used to show that $\operatorname{Fix}(\alpha) \cap \operatorname{Fix}(\beta)=\emptyset$.

\section{The SPace of Groups of type $(1,1)$ IN Möb $\left(\hat{\mathbb{R}}^{3}\right)$}

We define the space of Kleinian groups of type $(1,1)$ in $\operatorname{Möb}\left(\hat{\mathbb{R}}^{3}\right)$ by

$$
\widehat{\mathcal{M}}_{1,1}=\left\{\boldsymbol{p}=(p, q, r) \in \mathbb{R}^{3}: G_{\boldsymbol{p}}=\left\langle A_{\boldsymbol{p}}, B\right\rangle \text { is a rank-2 free Kleinian group }\right\} .
$$

The aim of this section is to study the shape of $\widehat{\mathcal{M}}_{1,1} \subset \mathbb{R}^{3}$. In particular, the slices of $\widehat{\mathcal{M}}_{1,1}$ along the planes $P_{q=0}$ and $P_{p=0}$ will be studied.

Remark. Although $B,\left[A_{\boldsymbol{p}}, B\right] \in G_{\boldsymbol{p}}$ are pure parabolic for every $\boldsymbol{p}$, the possibility remains that for some $\boldsymbol{p} \in \widehat{\mathcal{M}}_{1,1}$, the group $G_{\boldsymbol{p}}$ has (accidental) screw parabolic transformations. We do not know whether it does happen or not.

Observe that by definition we have

$$
\widehat{\mathcal{M}}_{1,1} \cap P_{r=0}=\mathcal{M}_{1,1}
$$

where the set $\mathcal{M}_{1,1} \subset \mathbb{C}$ is regarded as a subset of the plane $P_{r=0}$ via the identification $\mathbb{C} \ni p+i q \mapsto(p, q, 0) \in P_{r=0}$. 
We next observe in Theorem 5.1 below that there are two subsets $V_{1}, V_{2}$ of the parameter space $\mathbb{R}^{3}=\{\boldsymbol{p}=(p, q, r)\}$ such that $V_{1} \subset \widehat{\mathcal{M}}_{1,1} \subset V_{2}$ and that both of them are invariant under the action of the rotation along the $p$-axis. Recall that $R_{\theta} \in S O(3) \subset \operatorname{Möb}\left(\hat{\mathbb{R}}^{3}\right)$ denotes the rotation of angle $\theta \in \mathbb{R}$ around the $x$ axis. We also note that $R_{\theta}$ acts on the parameter space $\mathbb{R}^{3}=\{\boldsymbol{p}=(p, q, r)\}$ as a rotation around the $p$-axis. Furthermore, identifying $\hat{\mathbb{C}}$ with $\hat{P}_{r=0} \subset \hat{\mathbb{R}}^{3}$ via the map $\mu=p+i q \mapsto \boldsymbol{p}=(p, q, 0)$, we define a map $R_{\theta}: \hat{\mathbb{C}} \rightarrow \hat{\mathbb{R}}^{3}$ by

$$
R_{\theta}(\mu):=R_{\theta}(\boldsymbol{p})=(p, q \cos \theta, q \sin \theta) .
$$

Theorem 5.1. We have

$$
\bigsqcup_{0 \leq \theta<\pi} R_{\theta}(\{\mu \in \mathbb{C}:|\operatorname{Im} \mu| \geq 2\}) \subset \widehat{\mathcal{M}}_{1,1} \subset \bigsqcup_{0 \leq \theta<\pi} R_{\theta}\left(\mathcal{M}_{0,4}\right) .
$$

Proof. Suppose first that $\boldsymbol{p} \in \widehat{\mathcal{M}}_{1,1}$. Then $G_{\boldsymbol{p}}$ is discrete, and thus the subgroup $H_{\boldsymbol{p}} \subset G_{\boldsymbol{p}}$ is also discrete. Choose $0 \leq \theta<\pi$ and $\mu \in \mathbb{C}$ such that $\boldsymbol{p}=R_{\theta}(\mu)$. Then we have $H_{\boldsymbol{p}}=R_{\theta} H_{\mu} R_{\theta}^{-1}$, where $H_{\mu}$ is naturally regarded as a subgroup of $\operatorname{Möb}\left(\hat{\mathbb{R}}^{3}\right)$. It then follows from the discreteness of $H_{p}$ that $\mu \in \mathcal{M}_{0,4}$, and hence that $\boldsymbol{p}=R_{\theta}(\mu) \in \bigsqcup_{0 \leq \theta<\pi} R_{\theta}\left(\mathcal{M}_{0,4}\right)$.

Next suppose that $\boldsymbol{p} \in R_{\theta}(\{\mu \in \mathbb{C}:|\operatorname{Im} \mu| \geq 2\})$ for some $0 \leq \theta<\pi$. Then the interiors of the isometric spheres $I\left(A_{\boldsymbol{p}}\right)=\left\{\boldsymbol{x} \in \mathbb{R}^{3}:|\boldsymbol{x}|=1\right\}$ of $A_{\boldsymbol{p}}$ and $I\left(A_{\boldsymbol{p}}^{-1}\right)=\left\{\boldsymbol{x} \in \mathbb{R}^{3}:|\boldsymbol{x}-\boldsymbol{p}|=1\right\}$ of $A_{\boldsymbol{p}}^{-1}$ are disjoint. Therefore we can find a fundamental domain for $G_{\boldsymbol{p}}=\left\langle A_{\boldsymbol{p}}, B\right\rangle$ in $\hat{\mathbb{R}}^{3}$ as a 3 -dimensional analogue of the fundamental domain for $G_{\mu}$ in $\hat{\mathbb{C}}$ as in Figure 4. Then by Poincaré's polyhedron theorem (see Maskit [12, IV, H] and the remark below), $G_{\boldsymbol{p}}$ is a rank-2 free Kleinian group in $\operatorname{Möb}\left(\hat{\mathbb{R}}^{3}\right)$. Thus we conclude that $\boldsymbol{p} \in \widehat{\mathcal{M}}_{1,1}$.

Remark. Poincaré's polyhedron theorem for 3-dimensional polyhedra in $\hat{\mathbb{R}}^{3}$ with side-pairing maps in Möb $\left(\hat{\mathbb{R}}^{3}\right)$ can be deduced from the theorem for 4-dimensional polyhedra in $\mathbf{H}^{4}$ with side-pairing maps in $\operatorname{Isom}\left(\mathbf{H}^{4}\right)$; the precise statement can be found in Maskit [12]. We remark that, in general, a polyhedron in $\hat{\mathbb{R}}^{3}$ need not be convex; see also Epstein-Petronio [6].

5.1. Slice through the plane $P_{q=0}$. The goal of this subsection is to show Theorem 5.4, which states that the Maskit slice $\mathcal{M}_{0,4}$ of groups of type $(0,4)$ in $\operatorname{Möb}\left(\hat{\mathbb{R}}^{2}\right)$ appears as the slice of $\widehat{\mathcal{M}}_{1,1}$ through the plane $P_{q=0}=R_{\frac{\pi}{2}}(\mathbb{C})$.

Before starting the proof of Theorem 5.4, we will make some observations on groups $G_{\boldsymbol{p}} \subset \operatorname{Möb}\left(\hat{\mathbb{R}}^{3}\right)$ with $\boldsymbol{p} \in P_{q=0}$. Observe that if $\boldsymbol{p} \in P_{q=0}$, the action of $G_{\boldsymbol{p}}=\left\langle A_{\boldsymbol{p}}, B\right\rangle$ preserves the sphere $\hat{P}_{y=0}$. More precisely, let $\boldsymbol{p}=(p, 0, r) \in P_{q=0}$ and $\mu=p+i r \in \mathbb{C}$. Then the action of $G_{\boldsymbol{p}}=\left\langle A_{\boldsymbol{p}}, B\right\rangle$ restricted to the sphere $\hat{P}_{y=0}$ is given by

$$
\check{G}_{\mu}=\left\langle\check{A}_{\mu}, B\right\rangle ; \quad \check{A}_{\mu}(\tau)=\frac{1}{\bar{\tau}}+\mu, B(\tau)=\tau+2,
$$

where $\hat{P}_{y=0}$ is identified with $\hat{\mathbb{C}}$ via the map $\boldsymbol{x}=(x, 0, z) \mapsto \tau=x+i z$. (Here we use the notation $\check{G}_{\mu}$ and $\check{A}_{\mu}$ to distinguish them from $G_{\mu}$ and $A_{\mu}$ defined in 3.3.) Note that $\check{A}_{\mu}$ is an orientation reversing conformal automorphism of $\hat{\mathbb{C}}$ and that $\check{A}_{\mu}^{2}=A_{\mu}^{2}$. Let $\check{G}_{\mu}^{+}$be the index two subgroup of $\check{G}_{\mu}$ of orientation preserving transformations. Then we have

$$
\check{G}_{\mu}^{+}=\left\langle\check{A}_{\mu}^{2}, \check{A}_{\mu}^{-1} B \check{A}_{\mu}, B\right\rangle
$$


in fact, one can check that $\check{G}_{\mu}^{+} \subset \operatorname{Möb}\left(\hat{\mathbb{R}}^{2}\right)$ and $\left[\check{G}_{\mu}: \check{G}_{\mu}^{+}\right]=2$. Now observe that

and

$$
\check{A}_{\mu}^{-1} B \check{A}_{\mu}(\tau)=\frac{\tau}{2 \tau+1}=C(\tau)
$$

$$
\check{A}_{\mu} B \check{A}_{\mu}^{-1}(\tau)=D_{\mu}(\tau)=C(\tau-\mu)+\mu .
$$

It then follows that $H_{\mu}=\left\langle B, C, D_{\mu}\right\rangle$ is a subgroup of $\check{G}_{\mu}^{+}=\left\langle B, C, \check{A}_{\mu}^{2}\right\rangle$ and that $\check{G}_{\mu}^{+}=\left\langle H_{\mu}, \check{A}_{\mu}^{2}\right\rangle$. The statement of Theorem 5.4 can be rephrased so that $\check{G}_{\mu}^{+}$is discrete provided that $H_{\mu}$ is discrete.

To prove Theorem 5.4, we need to recall some basic facts of the action of a group $H_{\mu}=\left\langle B, C, D_{\mu}\right\rangle$ for $\mu \in \mathcal{M}_{0,4}$ on $\hat{\mathbb{C}}$. For simplicity we assume that $\operatorname{Im} \mu>0$, but the argument for the case $\operatorname{Im} \mu<0$ is parallel. Let $J_{1}=\langle B, C\rangle$ and $J_{2}=\left\langle B, D_{\mu}\right\rangle$ be the subgroups of $H_{\mu}$ of type $(0,3)$, and let $\Delta_{1}=\{\tau \in \mathbb{C}: \operatorname{Im} \tau<0\}$ and $\Delta_{2}=\{\tau \in \mathbb{C}: \operatorname{Im} \tau>\operatorname{Im} \mu\}$. In this notation, we have the following:

Lemma 5.2 (cf. [9]). (1) Let $i=1,2$. The disc $\Delta_{i}$ is a component of $\Omega\left(H_{\mu}\right)$, and is $\left(H_{\mu}, J_{i}\right)$-invariant; that is, $h\left(\Delta_{i}\right)=\Delta_{i}$ for every $h \in J_{i}$ and $h\left(\Delta_{i}\right) \cap$ $\Delta_{i}=\emptyset$ for every $h \in H \backslash J_{i}$.

(2) $h\left(\Delta_{1}\right) \cap \Delta_{2}=\emptyset$ for every $h \in H_{\mu}$.

(3) The set $\Omega_{0}\left(H_{\mu}\right):=\Omega\left(H_{\mu}\right) \backslash \bigcup_{h \in H_{\mu}} h\left(\Delta_{1} \cup \Delta_{2}\right)$ is either an empty set or a connected component of $\Omega\left(H_{\mu}\right)$ which is $H_{\mu}$-invariant and simply connected.

We state below the second Klein-Maskit combination theorem for Kleinian groups in $\operatorname{Möb}\left(\hat{\mathbb{R}}^{3}\right)$ to what extent we need in the proof of Theorem 5.4 (and its extension, Theorem 5.7). We refer the reader to Maskit [12, VII] for more information.

Theorem 5.3 (the second Klein-Maskit combination theorem [12]). Let $H \subset$ $\operatorname{Möb}\left(\hat{\mathbb{R}}^{3}\right)$ be a torsion-free Kleinian group and $A \in \operatorname{Möb}\left(\hat{\mathbb{R}}^{3}\right)$. Let $J_{1}, J_{2}$ be subgroups of $H$, and let $\mathbf{B}_{1}, \mathbf{B}_{2} \subset \hat{\mathbb{R}}^{3}$ be closed topological balls. If they satisfy the following conditions (1)-(5), then $G=\langle H, A\rangle$ is discrete and isomorphic to the $H N N$-extension $H *_{A}$ of $H$ by $A$ :

(1) Let $i=1,2$. The interior $\stackrel{\circ}{\mathbf{B}}_{i}$ of $\mathbf{B}_{i}$ is $\left(H, J_{i}\right)$-invariant; that is, $h\left(\stackrel{\circ}{\mathbf{B}}_{i}\right)=\stackrel{\circ}{\mathbf{B}}_{i}$ for every $h \in J_{i}$ and $h\left(\stackrel{\circ}{\mathbf{B}}_{i}\right) \cap \stackrel{\circ}{\mathbf{B}}_{i}=\emptyset$ for every $h \in H \backslash J_{i}$.

(2) $h\left(\stackrel{\circ}{\mathbf{B}}_{1}\right) \cap \stackrel{\circ}{\mathbf{B}}_{2}=\emptyset$ for every $h \in H$.

(3) The complement $\hat{\mathbb{R}}^{3} \backslash \bigcup_{h \in H} h\left(\mathbf{B}_{1} \cup \mathbf{B}_{2}\right)$ has an interior point.

(4) The transformation $A$ takes the interior of $\mathbf{B}_{1}$ onto the exterior of $\mathbf{B}_{2}$; that is, $A\left(\stackrel{\circ}{B}_{1}\right) \cap \stackrel{\circ}{\mathbf{B}}_{2}=\emptyset$ and $A\left(\partial \mathbf{B}_{1}\right)=\partial \mathbf{B}_{2}$.

(5) $J_{2}=A J_{1} A^{-1}$.

Remark. The conditions (1), (2) and (3) guarantee that $\stackrel{\circ}{\mathbf{B}}_{1} / J_{1}$ and $\stackrel{\circ}{\mathbf{B}}_{2} / J_{2}$ can be embedded disjointly into $\Omega(H) / H$, and that the complement $\Omega(H) / H \backslash$ $\left(\stackrel{\circ}{\mathbf{B}}_{1} / J_{1} \cup \stackrel{\circ}{\mathbf{B}}_{2} / J_{2}\right)$ has an interior point. The conditions (4) and (5) guarantee that the action of $A$ descends to a pairing map of the resulting boundary of $\Omega(H) / H \backslash\left(\stackrel{\circ}{\mathbf{B}}_{1} / J_{1} \cup \stackrel{\circ}{\mathbf{B}}_{2} / J_{2}\right)$.

For the convenience of the reader, we give a sketch of the proof of Theorem 5.3; see [12, VII. D and E] for more details.

Sketch of proof of Theorem 5.3. Recall from [12] that the group $H *_{A}$ is the free group of words in $A$ and the elements of $H$ modulo equivalence induced from 
the relation $J_{2}=A J_{1} A^{-1}$. Therefore each element of $H *_{A}$ is equivalent to a word of the form $A^{\alpha_{n}} h_{n} \cdots A^{\alpha_{1}} h_{1}\left(\alpha_{i} \in \mathbb{Z}, h_{i} \in H\right)$ which satisfy the following conditions: (1) $h_{i} \neq$ id for $i>1,(2) \alpha_{i} \neq 0$ for $i<n$, (3) $\alpha_{i+1}<0$ if $\alpha_{i}<0$ and $h_{i+1} \in J_{1} \backslash\{$ id $\}$, and (4) $\alpha_{i+1}>0$ if $\alpha_{i}>0$ and $h_{i+1} \in J_{2} \backslash\{$ id $\}$. We denote by $\Phi: H *_{A} \rightarrow\langle H, A\rangle \subset \operatorname{Möb}\left(\hat{\mathbb{R}}^{3}\right) ; \Phi\left(A^{\alpha_{n}} h_{n} \cdots A^{\alpha_{1}} h_{1}\right)=A^{\alpha_{n}} h_{n} \cdots A^{\alpha_{1}} h_{1}$ the natural projection.

Now let us take an open set $U$ in $\hat{\mathbb{R}}^{3} \backslash \bigcup_{h \in H} h\left(\mathbf{B}_{1} \cup \mathbf{B}_{2}\right)$. Since $H$ is a torsion-free Kleinian group, we may assume that $h(U) \cap U=\emptyset$ for every $h \in H \backslash\{$ id $\}$. We will show that $\Phi(g)(U) \cap U=\emptyset$ for every $g=A^{\alpha_{n}} h_{n} \cdots A^{\alpha_{1}} h_{1} \neq$ id. This implies that $\Phi$ is an isomorphism and $\langle H, A\rangle$ is discrete.

We first assume that $n=1$. If $\alpha_{1}=0$, then $g=h_{1} \in H \backslash\{\operatorname{id}\}$ and thus $g(U) \cap U=\emptyset$. When $\alpha_{1} \neq 0, g(U)=A^{\alpha_{1}} h_{1}(U)$ lies in $\stackrel{\mathbf{B}}{1}_{1}$ if $\alpha_{1}<0$ and in $\stackrel{\circ}{\mathbf{B}}_{2}$ if $\alpha_{1}>0$. In both cases we have $g(U) \cap U=\emptyset$.

Next assume that $n=2$. If $\alpha_{2}=0$, then $g=h_{2} A^{\alpha_{1}} h_{1}$ and $g(U) \subset$ $\bigcup_{h \in H} h\left(\stackrel{\circ}{\mathbf{B}}_{1} \cup \stackrel{\circ}{\mathbf{B}}_{2}\right)$, which implies that $g(U) \cap U=\emptyset$. When $\alpha_{2} \neq 0$, we can show that $g(U)=A^{\alpha_{2}} h_{2} A^{\alpha_{1}} h_{1}(U) \subset \stackrel{\circ}{\mathbf{B}}_{1} \cup \stackrel{\circ}{\mathbf{B}}_{2}$. In fact, if $h_{2} A^{\alpha_{1}} h_{1}(U) \subset \stackrel{\circ}{\mathbf{B}}_{1}$, one can see that $h_{2} \in J_{1}$ and $\alpha_{1}<0$. This implies that $\alpha_{2}<0$ and that $g(U) \subset \mathbf{B}_{1}$. Similarly, if $h_{2} A^{\alpha_{1}} h_{1}(U) \subset \stackrel{\circ}{\mathbf{B}}_{2}$ we have $g(U) \subset \stackrel{\circ}{\mathbf{B}}_{2}$. Finally, if $h_{2} A^{\alpha_{1}} h_{1}(U) \subset \hat{\mathbb{R}}^{3} \backslash\left(\stackrel{\circ}{\mathbf{B}}_{1} \cup \stackrel{\circ}{\mathbf{B}}_{2}\right)$, $g(U)$ lies in $\mathbf{B}_{1}$ if $\alpha_{2}<0$ and in $\stackrel{\circ}{\mathbf{B}}_{2}$ if $\alpha_{2}>0$. Thus we have $g(U) \subset \stackrel{\circ}{\mathbf{B}}_{1} \cup \stackrel{\circ}{\mathbf{B}}_{2}$, which implies that $g(U) \cap U=\emptyset$ also in this case.

The proof for the case of $n>2$ is obtained by induction.

Applying Lemma 5.2 and Theorem 5.3, we can now prove the following:

Theorem 5.4. We have

$$
\widehat{\mathcal{M}}_{1,1} \cap P_{q=0}=R_{\frac{\pi}{2}}\left(\mathcal{M}_{0,4}\right) .
$$

Proof. It follows from Theorem 5.1 that if $\boldsymbol{p} \in \widehat{\mathcal{M}}_{1,1} \cap P_{q=0}$, then $\boldsymbol{p} \in R_{\frac{\pi}{2}}\left(\mathcal{M}_{0,4}\right)$. Conversely, suppose that $\boldsymbol{p}=(p, 0, r) \in R_{\frac{\pi}{2}}\left(\mathcal{M}_{0,4}\right)$, and hence that the subgroup $H_{\boldsymbol{p}}=\left\langle B, C, D_{\boldsymbol{p}}\right\rangle$ of $G_{\boldsymbol{p}}$ is discrete. We assume for simplicity that the third coordinate $r$ of $\boldsymbol{p}=(p, 0, r)$ is positive, but the argument for the case $r<0$ is almost parallel. Let

$$
\begin{aligned}
& \mathbf{B}_{1}=\left\{(x, y, z) \in \mathbb{R}^{3}: z \leq 0\right\} \cup\{\infty\}, \\
& \mathbf{B}_{2}=\left\{(x, y, z) \in \mathbb{R}^{3}: z \geq r\right\} \cup\{\infty\}
\end{aligned}
$$

and

$$
J_{1}=\langle B, C\rangle, \quad J_{2}=\left\langle B, D_{\boldsymbol{p}}\right\rangle .
$$

We will check that $H_{\boldsymbol{p}}, A_{\boldsymbol{p}}, \mathbf{B}_{1}, \mathbf{B}_{2}, J_{1}$ and $J_{2}$ satisfy the conditions (1)-(5) in Theorem 5.3. Now take $\mu \in \mathcal{M}_{0,4}$ such that $\boldsymbol{p}=R_{\frac{\pi}{2}}(\mu)$. Then $\operatorname{Im} \mu>0$ by our assumption $r>0$. The map $R_{\frac{\pi}{2}}: \hat{\mathbb{C}} \rightarrow R_{\frac{\pi}{2}}(\hat{\mathbb{C}})=\hat{P}_{y=0}$ conjugates the action of $H_{\mu}$ on $\hat{\mathbb{C}}$ to the action of $H_{p}$ on the sphere $\hat{P}_{y=0}$. Therefore the group $H_{p}$ can be regarded as the Poincare extension of the group $H_{\mu}$ acting on the sphere $\hat{P}_{y=0} \cong \hat{\mathbb{C}}$ (see the left of Figure 6). Thus the conditions (1) and (2) directly follow from Lemma 5.2. Now observe that, for $i=1,2$, every orbit $h\left(\mathbf{B}_{i}\right)\left(h \in H_{\boldsymbol{p}}\right)$ of $\mathbf{B}_{i}$ except for $\mathbf{B}_{i}$ is a ball of radius $\leq r / 2$ with center in $P_{y=0}$. Therefore one can take an open domain, say, $\left\{(x, y, z) \in \mathbb{R}^{3}:|x|>r / 2,0<z<r\right\}$ which does not intersect $\bigcup_{h \in H} h\left(\mathbf{B}_{1} \cup \mathbf{B}_{2}\right)$. Thus the condition (3) follows. It is easy to 
check the condition (4). Finally, the condition (5) $J_{2}=A_{\boldsymbol{p}} J_{1} A_{\boldsymbol{p}}^{-1}$ follows from the facts that $B=A_{\boldsymbol{p}} C A_{\boldsymbol{p}}^{-1}$ and $D_{\boldsymbol{p}}=A_{\boldsymbol{p}} B A_{\boldsymbol{p}}^{-1}$. Therefore Theorem 5.3 asserts that $G_{\boldsymbol{p}}=\left\langle H_{\boldsymbol{p}}, A_{\boldsymbol{p}}\right\rangle$ is discrete and isomorphic to the group $H_{\boldsymbol{p}}{ }^{*} A_{\boldsymbol{p}}$. Thus we conclude that $\boldsymbol{p} \in \widehat{\mathcal{M}}_{1,1}$.
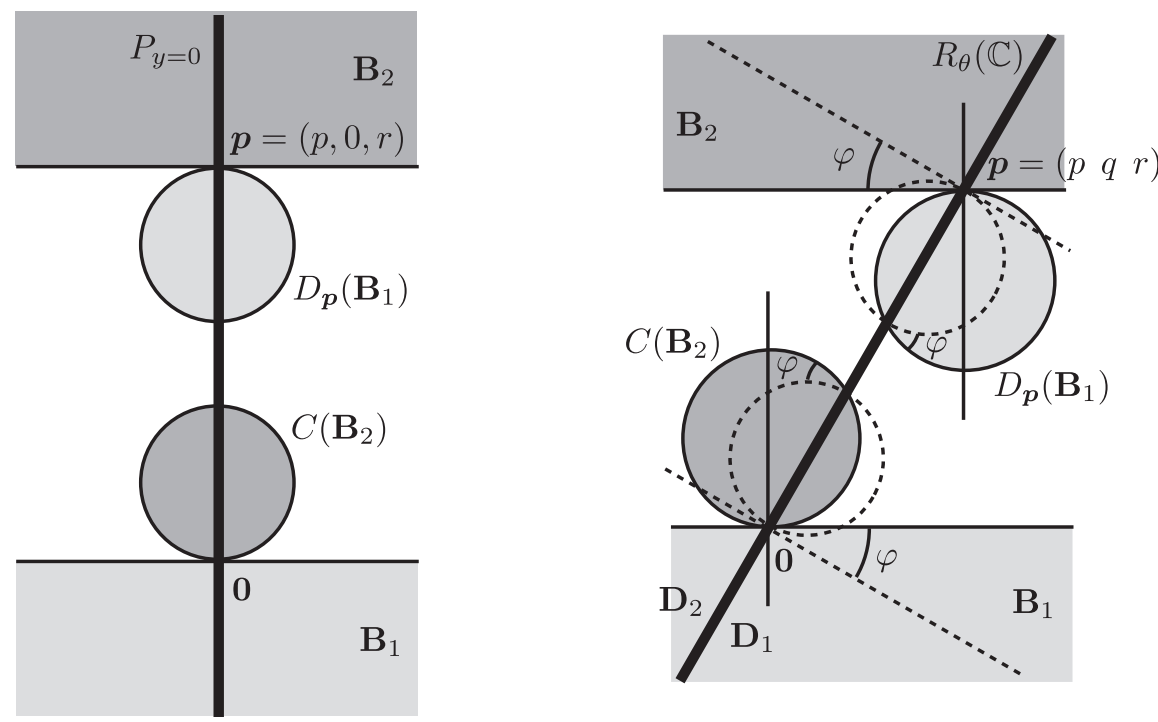

Figure 6 . The balls $\mathbf{B}_{1}, \mathbf{B}_{2}, C\left(\mathbf{B}_{2}\right)$ and $D_{\boldsymbol{p}}\left(\mathbf{B}_{1}\right)$ and the planes $P_{y=0}$ and $R_{\theta}(\mathbb{C})$ in the proofs of Theorems 5.4 (left) and 5.7 (right).

These are the views along the $x$-axis.

5.2. Slices through the planes $R_{\theta}(\mathbb{C})$. Recall from Theorem 5.4 that the Maskit slice $\mathcal{M}_{0,4}$ appears as the slice of $\widehat{\mathcal{M}}_{1,1}$ through the plane $P_{q=0}=R_{\frac{\pi}{2}}(\hat{\mathbb{C}})$. In this subsection, we will extend this result to Theorem 5.7, which states that there is a constant $\varphi_{0}>0$ such that $\mathcal{M}_{0,4}$ also appears as the slice of $\widehat{\mathcal{M}}_{1,1}$ through the plane $R_{\theta}(\hat{\mathbb{C}})$ for every $\theta \in\left[\pi / 2-\varphi_{0}, \pi / 2+\varphi_{0}\right]$.

We first fix our terminology:

Definition 5.5 (lens). The intersection $K_{1} \cap K_{2}$ of two spherical balls $K_{1}, K_{2}$ in $\hat{\mathbb{R}}^{3}$ is called a lens. The inner angle of lens $K_{1} \cap K_{2}$ is the interior angle $\psi(0<\psi<\pi)$ formed by two faces of $K_{1} \cap K_{2}$.

The following lemma is essential in the proof of Theorem 5.7; this is a special case of the collar lemma of Basmajian [3, Theorem 1.1].

Lemma 5.6 (Basmajian [3]). For a given $\mu \in \mathcal{M}_{0,4}$, let $\Delta$ be a non-invariant component of $\Omega\left(H_{\mu}\right)$. Let $\mathcal{C}(\Delta)$ be the convex hull of $\Delta$ in $\mathbf{H}^{3}$, and let $\operatorname{Stab}_{H_{\mu}}(\Delta)$ denote the stabilizer of $\Delta$ in $H_{\mu}$. Then there is a constant $k_{0}>0$, which does not depend on the choices of $\mu \in \mathcal{M}_{0,4}$ and $\Delta \subset \Omega\left(H_{\mu}\right)$, such that the $k_{0}$-neighborhood of $\mathcal{C}(\Delta)$ in $\mathbf{H}^{3}$ with respect to the hyperbolic metric is $\left(H_{\mu}, \operatorname{Stab}_{H_{\mu}}(\Delta)\right)$-invariant.

Remark. Note that $\mathcal{C}(\Delta) \subset \mathbf{H}^{3} \subset \hat{\mathbb{R}}^{3}$ can be regarded as a lens in $\hat{\mathbb{R}}^{3}$ with inner angle $\pi / 2$. Similarly, the $k_{0}$-neighborhood of $\mathcal{C}(\Delta)$ in $\mathbf{H}^{3}$ is a lens in $\hat{\mathbb{R}}^{3}$ with inner angle $\pi / 2+\varphi_{0}$, where $\varphi_{0}$ is the constant determined by $k_{0}$. 
We give here a proof of Lemma 5.6 for the convenience of the reader.

Proof of Lemma 5.6. Since $\Delta$ is a non-invariant component of $\Omega\left(H_{\mu}\right)$, it follows from Lemma 5.2 that the stabilizer $\operatorname{Stab}_{H_{\mu}}(\Delta)$ of $\Delta$ is a group of type $(0,3)$, and that $\Delta$ is $\left(H_{\mu}, \operatorname{Stab}_{H_{\mu}}(\Delta)\right)$-invariant. Therefore we only need to show that there exists a constant $k_{0}>0$, which does not depend on the choices of $\mu$ and $\Delta$, such that the hyperbolic distance from $\mathcal{C}(\Delta)$ to $h(\mathcal{C}(\Delta))$ is greater than $2 k_{0}$ for every $h \in H_{\mu} \backslash \operatorname{Stab}_{H_{\mu}}(\Delta)$.

Now let $\partial \mathcal{C}(\Delta)$ denote the relative boundary of $\mathcal{C}(\Delta)$ in $\mathbf{H}^{3}$ and let $r: \mathbf{H}^{3} \rightarrow$ $\partial \mathcal{C}(\Delta)$ be the nearest point retraction. For a given $h \in H_{\mu} \backslash \operatorname{Stab}_{H_{\mu}}(\Delta)$, let $U \subset \partial \mathcal{C}(\Delta)$ denote the image of $h(\mathcal{C}(\Delta))$ via the map $r$. Then one can check that $g(U) \cap U=\emptyset$ for every $g \in \operatorname{Stab}_{H_{\mu}}(\Delta)$. Therefore the hyperbolic area of $U$ is bounded above by the hyperbolic area $(=2 \pi)$ of the thrice-punctured sphere $\partial \mathcal{C}(\Delta) / \operatorname{Stab}_{H_{\mu}}(\Delta)$. From this, we can deduce the existence of a desired constant $k_{0}$.

Using Lemma 5.6, we can now prove the following:

Theorem 5.7. There is a constant $0<\varphi_{0}<\pi / 2$ such that

$$
\widehat{\mathcal{M}}_{1,1} \cap R_{\theta}(\mathbb{C})=R_{\theta}\left(\mathcal{M}_{0,4}\right)
$$

for every $\theta \in\left[\pi / 2-\varphi_{0}, \pi / 2+\varphi_{0}\right]$.

Proof. The argument is similar to the argument of the proof of Theorem 5.4. Let $0<\varphi_{0}<\pi / 2$ be the constant as in Remark of Lemma 5.6. We will show below that for every $\theta \in\left[\pi / 2-\varphi_{0}, \pi / 2+\varphi_{0}\right]$ we have $\widehat{\mathcal{M}}_{1,1} \cap R_{\theta}(\mathbb{C})=R_{\theta}\left(\mathcal{M}_{0,4}\right)$. By symmetry, we may assume that $\pi / 2-\varphi_{0} \leq \theta \leq \pi / 2$.

It follows from Theorem 5.1 that if $\boldsymbol{p} \in \widehat{\mathcal{M}}_{1,1} \cap R_{\theta}(\mathbb{C})$, then $\boldsymbol{p} \in R_{\theta}\left(\mathcal{M}_{0,4}\right)$. Conversely, suppose that $\boldsymbol{p}=(p, q, r) \in R_{\theta}\left(\mathcal{M}_{0,4}\right)$, and hence that the subgroup $H_{\boldsymbol{p}}=\left\langle B, C, D_{\boldsymbol{p}}\right\rangle$ of $G_{\boldsymbol{p}}$ is discrete. We again assume that $r>0$. Let $\mathbf{B}_{1}, \mathbf{B}_{2} \subset \hat{\mathbb{R}}^{3}$ and $J_{1}, J_{2} \subset H_{\boldsymbol{p}}$ be the same as in the proof of Theorem 5.4. We will check that $H_{\boldsymbol{p}}, A_{\boldsymbol{p}}, \mathbf{B}_{1}, \mathbf{B}_{2}, J_{1}$ and $J_{2}$ satisfy the conditions (1)-(5) in Theorem 5.3. Since the conditions (4) and (5) are similarly satisfied, we only need to check the conditions (1) $-(3)$.

Now take $\mu \in \mathcal{M}_{0,4}$ such that $R_{\theta}(\mu)=\boldsymbol{p}$. Then $\operatorname{Im} \mu>0$ by our assumption $r>0$. Observe that the action of group $H_{\boldsymbol{p}}$ preserves the sphere $R_{\theta}(\hat{\mathbb{C}})$, and that the map $R_{\theta}: \hat{\mathbb{C}} \rightarrow R_{\theta}(\hat{\mathbb{C}})$ conjugates the action of $H_{\mu}$ on $\hat{\mathbb{C}}$ to the action of $H_{\boldsymbol{p}}$ on the sphere $R_{\theta}(\hat{\mathbb{C}})$. Therefore the group $H_{\boldsymbol{p}}$ can be regarded as the Poincaré extension of the group $H_{\mu}$ acting on the sphere $R_{\theta}(\hat{\mathbb{C}}) \cong \hat{\mathbb{C}}$ (see the right of Figure $6)$. Observe that $R_{\theta}$ takes the components $\Delta_{1}, \Delta_{2}$ of $\Omega\left(H_{\mu}\right)$ as in Lemma 5.2 to the intersections

$$
\Delta_{1}^{\prime}:=\stackrel{\circ}{\mathbf{B}}_{1} \cap R_{\theta}(\hat{\mathbb{C}}) \quad \text { and } \quad \Delta_{2}^{\prime}:=\stackrel{\circ}{\mathbf{B}}_{2} \cap R_{\theta}(\hat{\mathbb{C}})
$$

of the balls $\stackrel{\circ}{\mathbf{B}}_{1}, \stackrel{\circ}{\mathbf{B}}_{2}$ with the sphere $R_{\theta}(\hat{\mathbb{C}})$, respectively. Therefore $\Delta_{i}^{\prime}$ is $\left(H_{\boldsymbol{p}}, J_{i}\right)$ invariant for $i=1,2$, and $h\left(\Delta_{1}^{\prime}\right) \cap \Delta_{2}^{\prime}=\emptyset$ for every $h \in H_{\boldsymbol{p}}$. Now let $\varphi$ denote $\pi / 2-\theta$. Then our assumption $\pi / 2-\varphi_{0} \leq \theta \leq \pi / 2$ can be written as $0 \leq \varphi \leq \varphi_{0}$. Let $\mathbf{D}_{1}, \mathbf{D}_{2}$ be the two components of $\hat{\mathbb{R}}^{3} \backslash R_{\theta}(\hat{\mathbb{C}})$ such that the inner angles of the lenses $\mathbf{D}_{1} \cap \mathbf{B}_{1}$ and $\mathbf{D}_{2} \cap \mathbf{B}_{1}$ are $\pi / 2+\varphi$ and $\pi / 2-\varphi$, respectively.

(1) We first show that $\stackrel{\circ}{\mathbf{B}}_{1}$ is $\left(H_{\boldsymbol{p}}, J_{1}\right)$-invariant. (The same argument reveals that $\mathbf{B}_{2}$ is $\left(H_{\boldsymbol{p}}, J_{2}\right)$-invariant. $)$ It is easy to see that $h\left(\stackrel{\circ}{\mathbf{B}}_{1}\right)=\stackrel{\circ}{\mathbf{B}}_{1}$ for every $h \in J_{1}$. 
To show that $\stackrel{\circ}{\mathbf{B}}_{1} \cap h\left(\stackrel{\circ}{\mathbf{B}}_{1}\right)=\emptyset$ for every $h \in H_{\boldsymbol{p}} \backslash J_{1}$, it suffices to show that $\left(\mathbf{D}_{1} \cap \stackrel{\circ}{\mathbf{B}}_{1}\right) \cap\left(\mathbf{D}_{1} \cap h\left(\stackrel{\mathbf{B}}{1}_{1}\right)\right)=\emptyset$. In fact, $h$ takes the lens $\mathbf{D}_{1} \cap \stackrel{\circ}{\mathbf{B}}_{1}$ with inner angle $\pi / 2+\varphi$ to the lens $\mathbf{D}_{1} \cap h\left(\stackrel{\mathbf{B}}{1}_{1}\right)$ with the same inner angle. Since we are assuming that $0 \leq \varphi \leq \varphi_{0}$, it follows from Lemma 5.6 that $\left(\mathbf{D}_{1} \cap \stackrel{\circ}{\circ}_{1}\right) \cap\left(\mathbf{D}_{1} \cap h\left(\stackrel{\circ}{\mathbf{B}}_{1}\right)\right)=\emptyset$.

(2) We next show that $h\left(\dot{\mathbf{B}}_{1}\right) \cap \dot{\mathbf{B}}_{2}=\emptyset$ for every $h \in H_{\boldsymbol{p}}$. By symmetry, it suffices to show that $\left(\mathbf{D}_{1} \cap h\left(\mathbf{B}_{1}\right)\right) \cap\left(\mathbf{D}_{1} \cap \mathbf{B}_{2}\right)=\emptyset$. This follows from the facts that $h\left(\Delta_{1}^{\prime}\right) \cap \Delta_{2}^{\prime}=\emptyset$, that the lens $\mathbf{D}_{1} \cap h\left(\mathbf{B}_{1}\right)$ has the inner angle $\pi / 2+\varphi$, and that the lens $\mathbf{D}_{1} \cap \mathbf{B}_{2}$ has the inner angle $\pi / 2-\varphi$.

(3) Finally, we show that $\hat{\mathbb{R}}^{3} \backslash \bigcup_{h \in H_{p}} h\left(\mathbf{B}_{1} \cup \mathbf{B}_{2}\right)$ has an interior point. This follows from the fact that, for $i=1,2$, every orbit $h\left(\mathbf{B}_{i}\right)\left(h \in H_{\boldsymbol{p}}\right)$ of $\mathbf{B}_{i}$ except for $\mathbf{B}_{i}$ is a ball of radius $\leq r / 2$ which intersects with the plane $R_{\theta}(\hat{\mathbb{C}})$.

Therefore Theorem 5.3 asserts that $G_{\boldsymbol{p}}=\left\langle H_{\boldsymbol{p}}, A_{\boldsymbol{p}}\right\rangle$ is discrete and isomorphic to the group $H_{p^{*} A_{p}}$. Thus we conclude that $\boldsymbol{p} \in \widehat{\mathcal{M}}_{1,1}$.

5.3. Slice through the plane $P_{p=0}$. In this subsection, we consider the case of $\boldsymbol{p} \in P_{p=0}$. In this case the cyclic subgroup $\left\langle A_{\boldsymbol{p}}\right\rangle \subset G_{\boldsymbol{p}}$ preserves the sphere $P_{x=0}$ and its orientation. One of the main results of this subsection is Theorem 5.9, which gives a necessary and sufficient condition for $\boldsymbol{p} \in P_{p=0}$ to be contained in $\widehat{\mathcal{M}}_{1,1}$. As a consequence, we will show in Corollary 5.11 that the boundary of the slice of $\widehat{\mathcal{M}}_{1,1}$ through the plane $P_{p=0}$ is a union of countably many analytic arcs, although the boundaries of the slices of $\widehat{\mathcal{M}}_{1,1}$ through the planes $P_{r=0}$ and $P_{q=0}$ are so-called "fractal."

We begin with the notion of a Ford domain: Let $\Gamma \subset \operatorname{Möb}\left(\hat{\mathbb{R}}^{3}\right)$ be a Kleinian group and suppose that $\gamma(\infty) \neq \infty$ for every $\gamma \in \Gamma \backslash\{$ id $\}$. Given $\gamma \in \Gamma$ we denote by $E(\gamma)$ the exterior of the isometric sphere $I(\gamma)$ of $\gamma$; that is, $E(\gamma)$ is the connected component of $\hat{\mathbb{R}}^{3} \backslash I(\gamma)$ containing $\infty$. Then the Ford domain for $\Gamma$ is defined by

$$
\operatorname{Ford}(\Gamma)=\bigcap_{\gamma \in \Gamma \backslash\{\mathrm{id}\}} E(\gamma),
$$

which turns out to be a fundamental domain for $\Gamma$. Below, we denote by radi $(I(f))$ the radius of the isometric sphere $I(f)$ of a transformation $f \in \operatorname{Möb}\left(\hat{\mathbb{R}}^{3}\right)$ with $f(\infty) \neq \infty$.

We will need the following lemma in the proof of Theorem 5.9.

Lemma 5.8. Let $f \in \operatorname{Möb}\left(\hat{\mathbb{R}}^{3}\right)$ be a loxodromic transformation with $f(\infty) \neq \infty$, and suppose that $f$ preserves the sphere $\hat{P}_{x=0}$ and its orientation. Let $B(\boldsymbol{x})=$ $\boldsymbol{x}+(2,0,0)$. Then $[f, B]$ is loxodromic, pure parabolic or elliptic if and only if $\operatorname{radi}(I(f))<1,=1$ or $>1$, respectively.

Proof. Note that the points $f^{-1}(\infty), f(\infty)$ lie in the plane $P_{x=0}$. We may assume that $f^{-1}(\infty)=\mathbf{0}$ after conjugating by a translation if necessary. We write $\boldsymbol{v}=$ $f(\infty)$. Then one see from 2.3 that $f(\boldsymbol{x})=P J_{I(f)}(\boldsymbol{x})+\boldsymbol{v}$ for some $P \in O(3) \backslash S O(3)$. Moreover, since $f$ preserves the sphere $\hat{P}_{x=0}$ and its orientation, one can see that $P\left(\boldsymbol{e}_{1}\right)=\boldsymbol{e}_{1}$, where $\boldsymbol{e}_{1}=(1,0,0)$.

Now write $r=\operatorname{radi}(I(f))$ and suppose first that $r=1$. Then we see that the transformation $[f, B]=f B f^{-1} B^{-1}$ maps the exterior of the sphere $S_{1}:=$ $B\left(I\left(f^{-1}\right)\right)$ onto the interior of the sphere $S_{2}$ of radius $1 / 4$ with center at $\boldsymbol{v}+3 / 4 \boldsymbol{e}_{1}$ (see Figure 7 ). Note that $S_{1}$ touches to $S_{2}$ at $\boldsymbol{v}+\boldsymbol{e}_{1}$, and that the transformation 
$[f, B]$ fixes the point $\boldsymbol{v}+\boldsymbol{e}_{1}$. Furthermore, we see that the differential of the map $[f, B]$ at this point is the identity. Thus we conclude that $[f, B]$ is pure parabolic. By the same argument, we see that if $r<1$, then $S_{1} \cap S_{2}=\emptyset$ and thus $[f, B]$ is loxodromic, and if $r>1$, then $[f, B]$ fixes every point of $S_{1} \cap S_{2}$ and thus $[f, B]$ is elliptic.

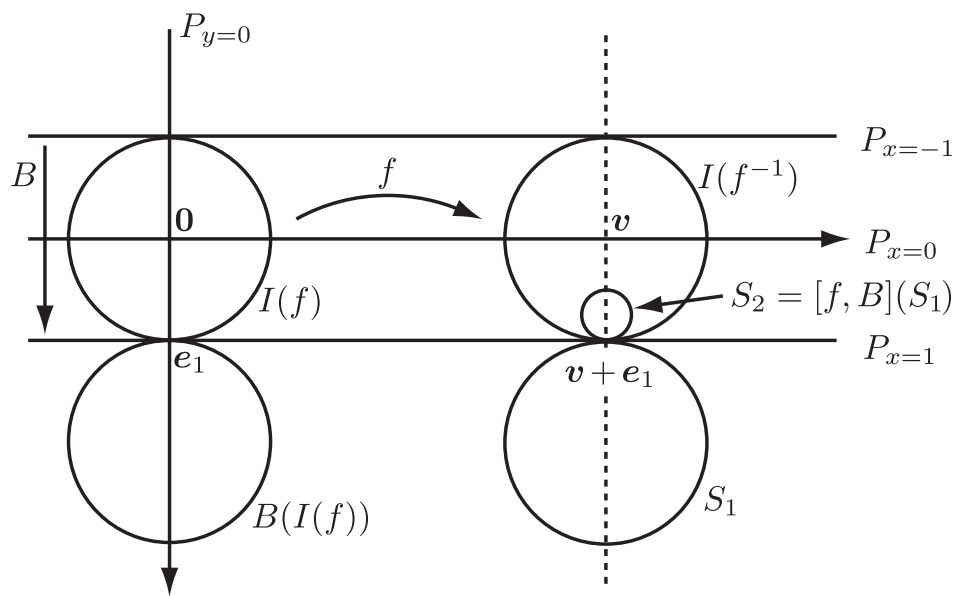

Figure 7. Surfaces $S_{1}$ and $S_{2}$ in the case of $r=1$. This is the view along the $z$-axis.

Theorem 5.9. Let $\boldsymbol{p} \in P_{p=0}$. Then $\boldsymbol{p} \in \widehat{\mathcal{M}}_{1,1}$ if and only if $\operatorname{radi}\left(I\left(A_{\boldsymbol{p}}^{n}\right)\right) \leq 1$ for all $n \in \mathbb{N}$. Moreover, for a given $n \in \mathbb{N}$, $\operatorname{radi}\left(I\left(A_{\boldsymbol{p}}^{n}\right)\right)=1$ if and only if $\left[A_{\boldsymbol{p}}^{n}, B\right]$ is pure parabolic.

Proof. Let $\boldsymbol{p} \in P_{p=0}$. We first remark that $A_{\boldsymbol{p}}^{n}(\infty) \neq \infty$ for every $n \in \mathbb{Z} \backslash\{0\}$, because $A_{\boldsymbol{p}}^{n}(\infty)$ lies in the interiors of $I\left(A_{\boldsymbol{p}}\right)$ or $I\left(A_{\boldsymbol{p}}^{-1}\right)$. Therefore the Ford domain

$$
\operatorname{Ford}\left(\left\langle A_{\boldsymbol{p}}\right\rangle\right)=\bigcap_{n \in \mathbb{Z} \backslash\{0\}} E\left(A_{\boldsymbol{p}}^{n}\right)
$$

for the cyclic group $\left\langle A_{\boldsymbol{p}}\right\rangle$ can be defined. In addition, observe that the center $A_{\boldsymbol{p}}^{n}(\infty)$ of $I\left(A_{\boldsymbol{p}}^{n}\right)$ lies in the plane $P_{x=0}$ for every $n \in \mathbb{Z} \backslash\{0\}$, and by definition that $\operatorname{radi}\left(I\left(A_{\boldsymbol{p}}^{n}\right)\right)=\operatorname{radi}\left(I\left(A_{\boldsymbol{p}}^{-n}\right)\right)$ for every $n \in \mathbb{N}$.

Suppose first that $\operatorname{radi}\left(I\left(A_{\boldsymbol{p}}^{n}\right)\right) \leq 1$ for all $n \in \mathbb{N}$. Then the Ford domain Ford $\left(\left\langle A_{\boldsymbol{p}}\right\rangle\right)$ contains the set $\left\{(x, y, z) \in \mathbb{R}^{3}:|x| \geq 1\right\}$. Therefore we can apply the Klein-Maskit combination theorem (Theorem 5.3) to show that $G_{\boldsymbol{p}}=\left\langle A_{\boldsymbol{p}}, B\right\rangle$ is discrete and isomorphic to the rank-2 free group $\left\langle A_{\boldsymbol{p}}\right\rangle *_{B}$ by letting $\mathbf{B}_{1}=\{(x, y, z) \in$ $\left.\mathbb{R}^{3}: x \leq-1\right\}, \mathbf{B}_{2}=\left\{(x, y, z) \in \mathbb{R}^{3}: x \geq 1\right\}$ and $J_{1}=J_{2}=\{\mathrm{id}\}$.

Conversely, suppose that $\operatorname{radi}\left(I\left(A_{\boldsymbol{p}}^{n}\right)\right)>1$ for some $n$. Then by Lemma 5.8, $\left[A_{\boldsymbol{p}}^{n}, B\right]$ is elliptic. If the order of this elliptic element is infinite, then the group $G_{p}$ is non-discrete, and if the order is finite, then $G_{p}$ is not free. In both cases, we conclude $\boldsymbol{p} \notin \widehat{\mathcal{M}}_{1,1}$.

The second statement also follows from Lemma 5.8. 
Now let $\boldsymbol{p}=(0, q, r) \in P_{p=0}$ and $\mu=q+i r \in \mathbb{C}$. In this notation, the map $A_{\boldsymbol{p}}(\boldsymbol{x})=\hat{J} J(\boldsymbol{x})+\boldsymbol{p}$ restricted to the sphere $\hat{P}_{x=0}$ is a Möbius transformation given by

$$
\dot{A}_{\mu}(\tau)=-\frac{1}{\tau}+\mu,
$$

where $\hat{P}_{x=0}$ is identified with $\hat{\mathbb{C}}$ via the map $\boldsymbol{x}=(0, y, z) \mapsto \tau=y+i z$. (Here we use the notation $\dot{A}_{\mu}$ to distinguish it from $A_{\mu}$ and $\check{A}_{\mu}$.) The matrix representation of $A_{\mu}$ is

$$
\dot{A}_{\mu}=\left(\begin{array}{cc}
\mu & -1 \\
1 & 0
\end{array}\right) \in \mathrm{PSL}_{2}(\mathbb{C})
$$

We denote the entries of $A_{\mu}^{n}$ for $n \in \mathbb{N}$ by

$$
\dot{A}_{\mu}^{n}=\left(\begin{array}{ll}
a_{n} & b_{n} \\
c_{n} & d_{n}
\end{array}\right) .
$$

Then the radius of the isometric sphere $I\left(\hat{A}_{\mu}^{n}\right)$ of $\dot{A}_{\mu}^{n}$ is given by $1 /\left|c_{n}\right|$. In other words, we have $\operatorname{radi}\left(I\left(A_{\boldsymbol{p}}^{n}\right)\right)=\operatorname{radi}\left(I\left(A_{\boldsymbol{p}}^{-n}\right)\right)=1 /\left|c_{n}\right|$ for every $n \in \mathbb{N}$. Since

$$
\begin{aligned}
\left(\begin{array}{cc}
a_{n+1} & b_{n+1} \\
c_{n+1} & d_{n+1}
\end{array}\right) & =\left(\begin{array}{cc}
\mu & -1 \\
1 & 0
\end{array}\right)\left(\begin{array}{cc}
a_{n} & b_{n} \\
c_{n} & d_{n}
\end{array}\right) \\
& =\left(\begin{array}{cc}
\mu a_{n}-c_{n} & \mu b_{n}-d_{n} \\
a_{n} & b_{n}
\end{array}\right),
\end{aligned}
$$

we have $a_{n+1}=\mu a_{n}-c_{n}$ and $c_{n+1}=a_{n}$. Therefore we obtain the following recurrence equations:

$$
c_{1}=1, \quad c_{2}=\mu \quad \text { and } \quad c_{n+2}=\mu c_{n+1}-c_{n}(n \in \mathbb{N}) .
$$

In particular, $c_{n}=c_{n}(\mu)$ is a monic $\mu$-polynomial of degree $(n-1)$; for example,

$$
\begin{aligned}
& c_{3}(\mu)=\mu^{2}-1, \\
& c_{4}(\mu)=\mu^{3}-2 \mu, \\
& c_{5}(\mu)=\mu^{4}-3 \mu^{2}+1,
\end{aligned}
$$

and so on. Using this notation and identifying $P_{p=0}$ with $\mathbb{C}$, we can rephrase Theorem 5.9 as follows.

Theorem 5.10. We have

$$
\widehat{\mathcal{M}}_{1,1} \cap P_{p=0}=\bigcap_{n \in \mathbb{N}}\left\{\mu \in \mathbb{C}:\left|c_{n}(\mu)\right| \geq 1\right\} .
$$

Since the set $\left\{\mu \in \mathbb{C}:\left|c_{n}(\mu)\right|=1\right\}$ is a one-dimensional real analytic variety for every $n \in \mathbb{N}$, we have the following corollary.

Corollary 5.11. The boundary of $\widehat{\mathcal{M}}_{1,1} \cap P_{p=0}$ in the plane $P_{p=0}$ is a union of countably many analytic arcs.

One can find in Figures 8 and 9 the numerical graphics of the loci of $\mu \in \mathbb{C}$ such that $\left|c_{n}(\mu)\right|=1$ or $\leq 1$ for some $n$. 


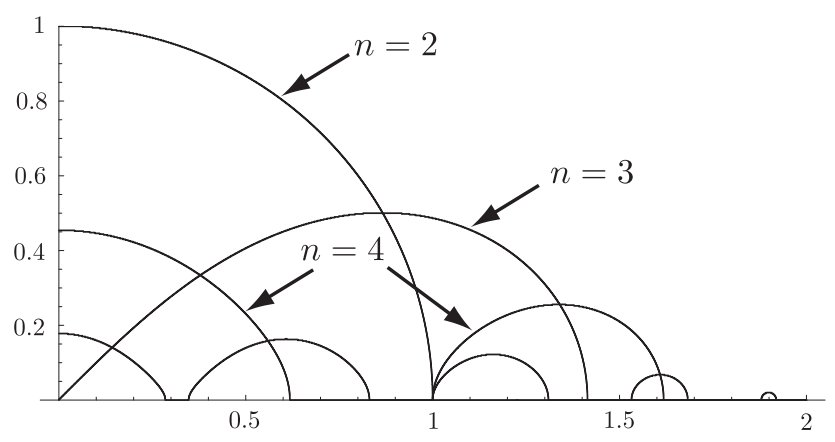

FiguRe 8. The loci of $\mu \in\{q+i r \in \mathbb{C}: 0 \leq q \leq 2,0 \leq r \leq 1\}$ such that $\left|c_{n}(\mu)\right|=1$ for $n=2,3,4$ and 10 . The arcs without labels correspond to $n=10$.

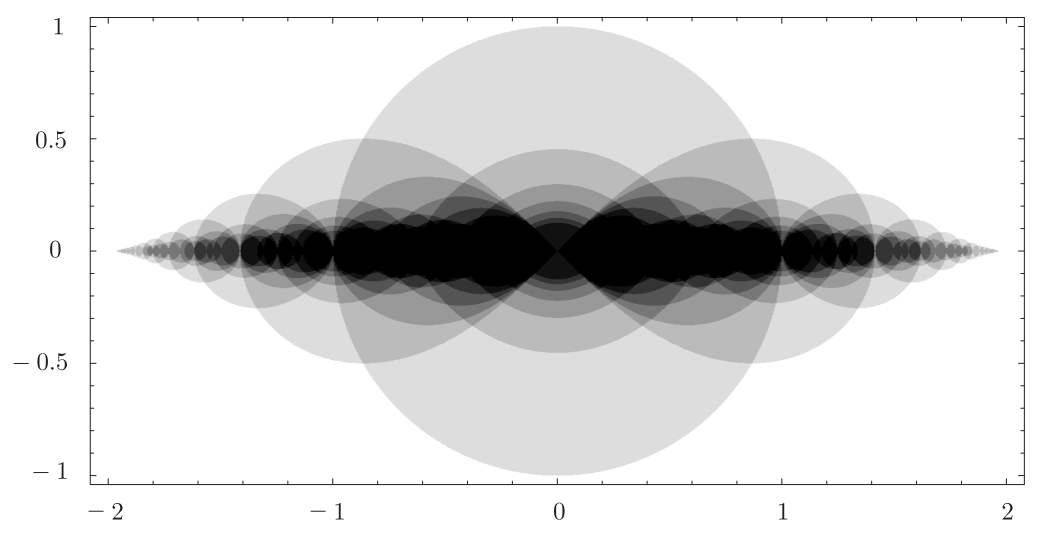

Figure 9. The union of the loci of $\mu \in\{q+i r \in \mathbb{C}:-2 \leq q \leq$ $2,-1 \leq r \leq 1\}$ such that $\left|c_{n}(\mu)\right| \leq 1$ for $2 \leq n \leq 20$. The darkness increases as the number of the intersections increases.

\section{ApPEndix}

In this section we consider a family of 3 -generator Kleinian groups in Möb $\left(\hat{\mathbb{R}}^{3}\right)$, which can be viewed as an analogue of the family of groups of type $(1,1)$ in $\operatorname{Möb}\left(\hat{\mathbb{R}}^{2}\right)$. These groups contain groups of type $(1,1)$ in $\operatorname{Möb}\left(\hat{\mathbb{R}}^{3}\right)$ and their limit sets are union of round spheres.

For a given $\boldsymbol{p}=(0, q, 0) \in \mathbb{R}^{3}$ with $q>2$, we define an ideal hexahedron $\mathcal{D}_{\boldsymbol{p}}$ as follows (see Figure 10):

$$
\mathcal{D}_{\boldsymbol{p}}=\left\{\begin{array}{ll}
\boldsymbol{x}=(x, y, z) \in \mathbb{R}^{3}: \begin{array}{l}
|x| \leq 1 / \sqrt{2}, 0 \leq y \leq q,|z| \leq 1 / \sqrt{2}, \\
|\boldsymbol{x}| \geq 1,|\boldsymbol{x}-\boldsymbol{p}| \geq 1
\end{array}
\end{array}\right\} .
$$

Observe that eight edges of $\mathcal{D}_{p}$ have dihedral angle $\pi / 4$ and the remaining four edges have dihedral angle $\pi / 2$. Moreover, the following three transformations in $\operatorname{Möb}\left(\hat{\mathbb{R}}^{3}\right)$ pair the faces of $\mathcal{D}_{\boldsymbol{p}}$ :

$$
A_{\boldsymbol{p}}(\boldsymbol{x})=\hat{J} J(\boldsymbol{x})+\boldsymbol{p}, B(\boldsymbol{x})=\boldsymbol{x}+(\sqrt{2}, 0,0), C(\boldsymbol{x})=\boldsymbol{x}+(0,0, \sqrt{2}) .
$$




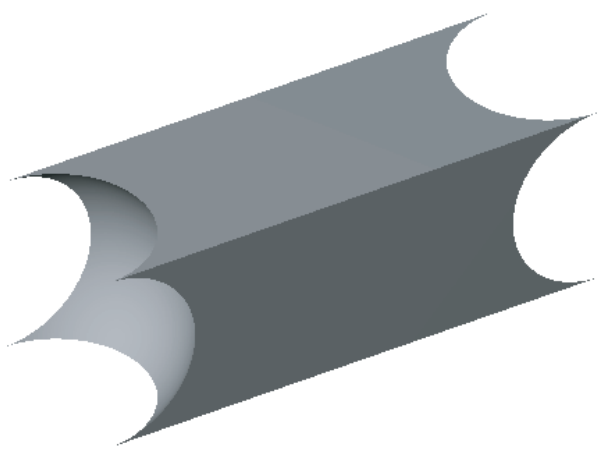

Figure 10 . The ideal hexahedron $\mathcal{D}_{\boldsymbol{p}}$ with $\boldsymbol{p}=(0,4,0)$.

(Although the notation $A_{p}$ is common with the previous sections, we remark that $B, C$ are different from those in the previous sections.) Therefore, it follows from Poincaré's polyhedron theorem (cf. [12) that the group

$$
K_{\boldsymbol{p}}=\left\langle A_{\boldsymbol{p}}, B, C\right\rangle
$$

generated by $A_{\boldsymbol{p}}, B$ and $C$ in $\operatorname{Möb}\left(\hat{\mathbb{R}}^{3}\right)$ is discrete, and that $K_{\boldsymbol{p}}$ is isomorphic to the abstract group

$$
\mathfrak{g}=\left\langle a, b, c:[a, b]^{2}=[a, c]^{2}=[b, c]=\mathrm{id}\right\rangle .
$$

For arbitrary $\boldsymbol{p} \in \mathbb{R}^{3}$, we also denote by $K_{\boldsymbol{p}}=\left\langle A_{\boldsymbol{p}}, B, C\right\rangle$ the group in $\operatorname{Möb}\left(\hat{\mathbb{R}}^{3}\right)$ generated by the transformations $A_{p}, B$ and $C$ as in (6.1). Note that if $K_{p}$ is isomorphic to $\mathfrak{g}$, the subgroup $\left\langle A_{\boldsymbol{p}}, B C\right\rangle$ of $K_{\boldsymbol{p}}$ is a group of type $(1,1)$ in $\operatorname{Möb}\left(\hat{\mathbb{R}}^{3}\right)$ because $B C(\boldsymbol{x})=\boldsymbol{x}+(\sqrt{2}, 0, \sqrt{2})$ is a translation of length 2 .

6.1. Groups of type $(1,0 ; 2)$. For a given $\boldsymbol{p}_{0}=(0, q, 0) \in \mathbb{R}^{3}$ with $q>2$, we will study deformations of $K_{\boldsymbol{p}_{0}}$ in the next subsection. As a preparation for this purpose, in this subsection, we study deformations of the subgroup $\left\langle A_{p_{0}}, B\right\rangle$ of $K_{p_{0}}=\left\langle A_{p_{0}}, B, C\right\rangle$.

Let $\Sigma_{1,0 ; 2}$ denote a torus with a singular point of cone-angle $\pi$. The fundamental group $\pi_{1}\left(\Sigma_{1,0 ; 2}\right)$ of the orbifold $\Sigma_{1,0 ; 2}$ is isomorphic to an abstract group $\langle a, b$ : $\left.[a, b]^{2}=\mathrm{id}\right\rangle$. We say that a group $\Gamma$ in $\operatorname{Möb}\left(\hat{\mathbb{R}}^{3}\right)$ is of type $(1,0 ; 2)$ if it is the image of a faithful representation $\rho: \pi_{1}\left(\Sigma_{1,0 ; 2}\right)=\left\langle a, b:[a, b]^{2}=\mathrm{id}\right\rangle \rightarrow \operatorname{Möb}\left(\hat{\mathbb{R}}^{3}\right)$ such that $\rho(b)$ is pure parabolic. Note that if $K_{p}=\left\langle A_{\boldsymbol{p}}, B, C\right\rangle$ is isomorphic to $\mathfrak{g}$, the subgroups $\left\langle A_{\boldsymbol{p}}, B\right\rangle,\left\langle A_{\boldsymbol{p}}, C\right\rangle$ of $K_{\boldsymbol{p}}$ are of type $(1,0 ; 2)$ in Möb $\left(\hat{\mathbb{R}}^{3}\right)$. Similar to Theorem 4.5 , we have the following normalization of groups of type $(1,0 ; 2)$ in $\operatorname{Möb}\left(\hat{\mathbb{R}}^{3}\right)$.

Theorem 6.1. Let $\Gamma=\langle\alpha, \beta\rangle$ be a subgroup of $\mathrm{Möb}\left(\hat{\mathbb{R}}^{3}\right)$ which is isomorphic to the abstract group $\left\langle a, b:[a, b]^{2}=\mathrm{id}\right\rangle$ and suppose that $\beta$ is pure parabolic. Then $\Gamma$ is conjugate in $\operatorname{Möb}\left(\hat{\mathbb{R}}^{3}\right)$ to $\left\langle A_{\boldsymbol{p}}, B\right\rangle$ for some $\boldsymbol{p}=(p, q, r) \in \mathbb{R}^{3}$, where $A_{\boldsymbol{p}}, B \in \operatorname{Möb}\left(\hat{\mathbb{R}}^{3}\right)$ are as in $(6.1)$. 
Proof. The proof is almost parallel to the proof of Theorem 4.5. We can first show that $\operatorname{Fix}(\alpha) \cap \operatorname{Fix}(\beta)=\emptyset$. In fact, if not, the same argument as in the proof of Theorem 4.5 implies that $[\beta,[\alpha, \beta]]=\mathrm{id}$, which contradicts the assumption that $\Gamma=\langle\alpha, \beta\rangle$ is isomorphic to the abstract group $\left\langle a, b:[a, b]^{2}=\mathrm{id}\right\rangle$. Therefore, by using the same normalization as in the proof of Theorem 4.5, we may assume that $\alpha, \beta$ are of the forms $\alpha(\boldsymbol{x})=P J(\boldsymbol{x})+\boldsymbol{p}, \beta(\boldsymbol{x})=\boldsymbol{x}+(u, v, 0)$ where $P \in O(3) \backslash S O(3)$, $\boldsymbol{p} \in \mathbb{R}^{3}$ and $u, v \geq 0$ such that $P^{-1}(\boldsymbol{u})=(u,-v, 0)$. Then $\alpha^{-1} \beta^{-1} \alpha \beta$ preserves the sphere $\hat{P}_{z=0} \cong \hat{\mathbb{C}}$ and its orientation. Furthermore, identifying $\hat{P}_{z=0}$ with $\hat{\mathbb{C}}$ via the map $(x, y, 0) \mapsto x+i y$ and letting $\mu=u+i v$, one sees that the map $\alpha^{-1} \beta^{-1} \alpha \beta$ restricted to the sphere $\hat{P}_{z=0} \cong \hat{\mathbb{C}}$ is a Möbius transformation whose matrix representation is

$$
\left(\begin{array}{cc}
1 & \mu \\
-\mu & 1-\mu^{2}
\end{array}\right) \in \mathrm{PSL}_{2}(\mathbb{C})
$$

It then follows from the condition $[\alpha, \beta]^{2}=$ id that $\mu=\sqrt{2}$, and hence, that $\boldsymbol{u}=P^{-1}(\boldsymbol{u})=(\sqrt{2}, 0,0)$. The remaining argument is the same as the argument of the proof of Theorem 4.5.

6.2. Deformations of the group $K_{\boldsymbol{p}}$. For a given $\boldsymbol{p}_{0}=(0, q, 0) \in \mathbb{R}^{3}$ with $q>2$, we now consider deformations $\left\{\phi: K_{\boldsymbol{p}_{0}} \rightarrow \Gamma\right\}$ of $K_{\boldsymbol{p}_{0}}$ in $\operatorname{Möb}\left(\hat{\mathbb{R}}^{3}\right)$ such that the isomorphism $\phi$ takes a pure parabolic transformation to a pure parabolic transformation. The following theorem reveals that every such deformation $\Gamma$ of $K_{\boldsymbol{p}_{0}}$ is conjugate to $K_{\boldsymbol{p}}$ for some $\boldsymbol{p} \in \mathbb{R}^{3}$.

Theorem 6.2. Let $\Gamma=\langle\alpha, \beta, \gamma\rangle$ be a subgroup of $\operatorname{Möb}\left(\hat{\mathbb{R}}^{3}\right)$ which is isomorphic to the abstract group $\mathfrak{g}=\left\langle a, b, c:[a, b]^{2}=[a, c]^{2}=[b, c]=\mathrm{id}\right\rangle$, and suppose that $\beta, \gamma$ and $[\alpha, \beta \gamma]$ are pure parabolic. Then $\Gamma$ is conjugate in $\operatorname{Möb}\left(\hat{\mathbb{R}}^{3}\right)$ to $K_{p}=\left\langle A_{p}, B, C\right\rangle$ for some $\boldsymbol{p} \in \mathbb{R}^{3}$.

Proof. Note that the subgroups $\langle\alpha, \beta\rangle,\langle\alpha, \gamma\rangle$ of $\Gamma$ are of type $(1,0 ; 2)$ in $\operatorname{Möb}\left(\hat{\mathbb{R}}^{3}\right)$, and $\langle\alpha, \beta \gamma\rangle$ is of type $(1,1)$. By Theorem 6.1 , we may assume that $\langle\alpha, \beta\rangle=\left\langle A_{p}, B\right\rangle$ for some $\boldsymbol{p} \in \mathbb{R}^{3}$ after conjugating $\langle\alpha, \beta, \gamma\rangle$ if necessary. We will show that $\gamma=C^{ \pm 1}$ below. Since $\gamma$ is pure parabolic and commutes with $B$, we have $\gamma(\infty)=\infty$. Since we are normalizing so that the radius of the isometric sphere of $\alpha=A_{p}$ equals 1 , it follows from Theorem 6.1 that $\gamma$ is a translation of length $\sqrt{2}$, and from Theorem 4.5 that $B \gamma$ is a translation of length 2. Therefore the direction of translations of $B$ and $\gamma$ are perpendicular. This implies that $\gamma=C^{ \pm 1}$. Thus we obtain $\langle\alpha, \beta, \gamma\rangle=\left\langle A_{\boldsymbol{p}}, B, C\right\rangle$.

We now define:

$$
\mathcal{N}=\left\{\boldsymbol{p}=(p, q, r) \in \mathbb{R}^{3}: K_{\boldsymbol{p}}=\left\langle A_{\boldsymbol{p}}, B, C\right\rangle \text { is discrete and } \cong \mathfrak{g}\right\} .
$$

Since the group $K_{\boldsymbol{p}}$ for $\boldsymbol{p} \in \mathcal{N}$ contains the groups $\left\langle A_{\boldsymbol{p}}, B^{-1} C\right\rangle,\left\langle A_{\boldsymbol{p}}, B C\right\rangle$ of type $(1,1)$ in $\operatorname{Möb}\left(\hat{\mathbb{R}}^{3}\right)$, the set $\mathcal{N} \subset \mathbb{R}^{3}$ lies in the rotations of the set $\widehat{\mathcal{M}}_{1,1}$ of angles $\pi / 4,3 \pi / 4$ along the $q$-axis. One can also see that $\mathcal{N}$ is invariant under the action of the translations $\boldsymbol{x} \mapsto \boldsymbol{x}+(\sqrt{2} m, 0, \sqrt{2} n), m, n \in \mathbb{Z}$. The following lemma shows that the set $\mathcal{N}$ contains a 3 -dimensional domain of $\mathbb{R}^{3}$.

Lemma 6.3. We have $\left\{\boldsymbol{p}=(p, q, r) \in \mathbb{R}^{3}:|q| \geq 2\right\} \subset \mathcal{N}$. 
Proof. For a given $\boldsymbol{p}=(p, q, r) \in \mathbb{R}^{3}$ with $|q| \geq 2$, we let $\boldsymbol{p}_{0}=(0, q, 0)$. We suppose that $q \geq 2$ for simplicity, but the argument for the case of $q \leq-2$ is almost parallel. Deforming the ideal hexahedron $\mathcal{D}_{\boldsymbol{p}_{0}}$ in a similar way as in Figure 4 , we can obtain an ideal polyhedron to which Poincaré's polyhedron theorem can be applied (cf. [12]) to show that the group $K_{p}=\left\langle A_{p}, B, C\right\rangle$ is discrete and isomorphic to the abstract group $\mathfrak{g}$. Thus we conclude that $\boldsymbol{p} \in \mathcal{N}$.

6.3. Limit sets of Kleinian groups $K_{p}$. For a given $\boldsymbol{p} \in \mathbb{R}^{3}$, one can see that $A_{p}^{-1} B A_{p}=J B J$ and $A_{p}^{-1} C A_{p}=J C J$ hold. Therefore the group $K_{p}=\left\langle A_{p}, B, C\right\rangle$ always contains the group

$$
L:=\langle B, C, J B J, J C J\rangle
$$

Note that the action of $L$ preserves the sphere $\hat{P}_{y=0}$ and its orientation. Now we take an ideal pentahedron $\mathcal{D}^{-}$as follows:

$$
\mathcal{D}^{-}=\left\{\boldsymbol{x}=(x, y, z) \in \mathbb{R}^{3}:|x| \leq 1 / \sqrt{2},|z| \leq 1 / \sqrt{2},|\boldsymbol{x}| \geq 1, y \leq 0\right\} .
$$

Then one can see that the ideal octahedron $\mathcal{D}^{-} \cup J\left(\mathcal{D}^{-}\right)$is a fundamental domain for the action of $L$ acting on the half-space $\left\{(x, y, z) \in \mathbb{R}^{3}: y<0\right\}$. Therefore $L$ can be regarded as a Kleinian group in $\operatorname{Möb}\left(\hat{\mathbb{R}}^{2}\right)$ of the first-kind; that is, the limit set $\Lambda(L)$ of $L$ is the whole sphere $\hat{P}_{y=0}$. Thus the limit set $\Lambda\left(K_{p}\right)$ of a Kleinian group $K_{p}$ for $\boldsymbol{p} \in \mathcal{N}$ is the closure of a union of round spheres:

$$
\Lambda\left(K_{\boldsymbol{p}}\right)=\overline{\bigcup_{\gamma \in K_{\boldsymbol{p}} / L} \gamma(\Lambda(L)) .}
$$

One can find in Figure 11 some computer graphics of the limit sets of $K_{p}$ for parameters $\boldsymbol{p} \in \mathbb{R}^{3}$ which lie (or seem to lie) in $\mathcal{N}$.

By using the observation of the subgroup $L \subset K_{p}$ above, we obtain an alternative proof of Lemma 6.3. In fact, a Kleinian group $K_{\boldsymbol{p}}$ for $\boldsymbol{p}=(p, q, r)$ with $|q| \geq 2$ can be regarded as an amalgamation of the Kleinian group $L$ with the cyclic group $\left\langle A_{\boldsymbol{p}}\right\rangle$ as follows: Suppose $q \geq 2$ for simplicity. Then we can apply the second KleinMaskit combination theorem (Theorem 5.3) to show that $K_{p}=\left\langle L, A_{p}\right\rangle$ is discrete and $K_{\boldsymbol{p}} \cong L *_{A_{p}}$ by letting

$$
\begin{aligned}
& \mathbf{B}_{1}=\left\{\boldsymbol{x} \in \mathbb{R}^{3}:\left|\boldsymbol{x}-\left(0, \frac{q}{4}, 0\right)\right| \leq \frac{q}{4}\right\}, \\
& \mathbf{B}_{2}=\left\{(x, y, z) \in \mathbb{R}^{3}: y \geq q-\frac{2}{q}\right\},
\end{aligned}
$$

$J_{1}=\langle J B J, J C J\rangle$ and $J_{2}=\langle B, C\rangle$.

\section{ACKNOWLEDGMENTS}

The authors would like to thank Shin Nayatani and Yasushi Yamashita for their interest and useful comments, Yohei Komori for his valuable information and discussions, and Keita Sakugawa for bringing to our attention to deformations of Kleinian groups with screw parabolic transformations. We are also grateful for the referee's valuable comments and suggestions. 


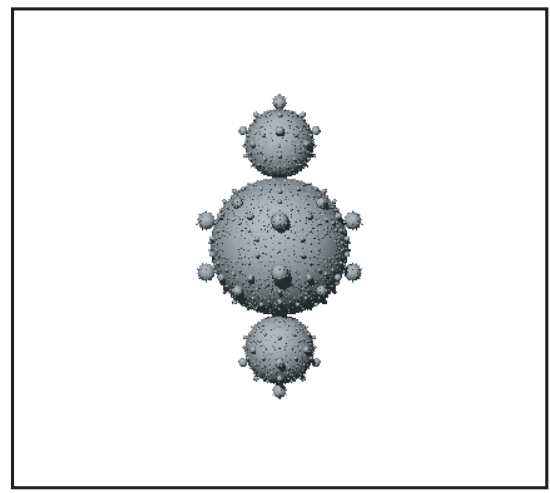

$(p, q, r)=(0,3,0)$

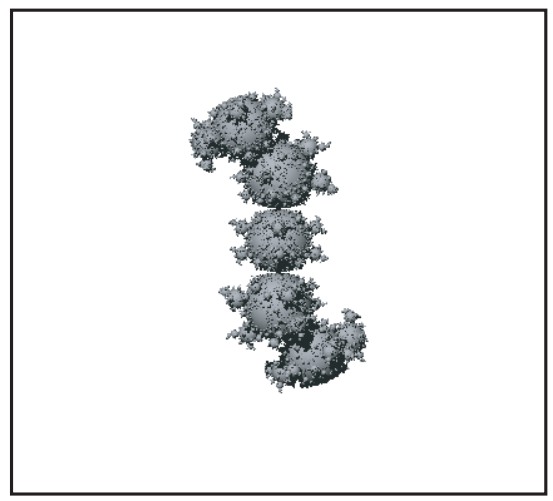

$(p, q, r)=(0.3,1.95,0.2)$

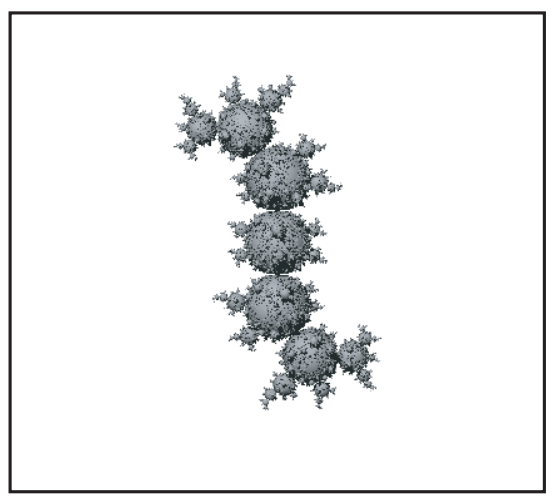

$(p, q, r)=(0.3,2,0)$

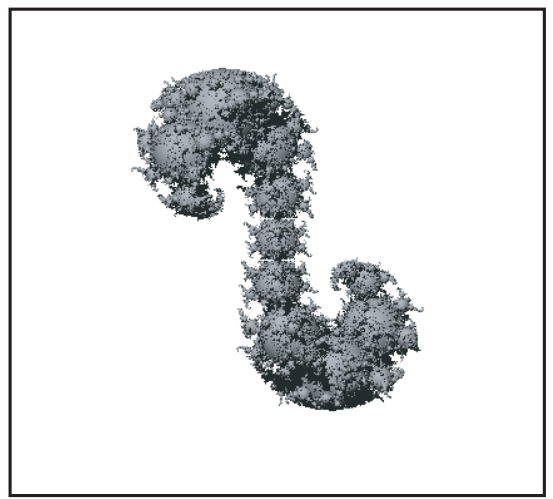

$(p, q, r)=(0.1,1.9,0.1)$

Figure 11. Computer graphics of the limit sets $\Lambda\left(K_{p}\right)$ of groups $K_{p}$ : These are the images $f\left(\Lambda\left(K_{p}\right)\right)$ of $\Lambda\left(K_{p}\right)$ by the Möbius transformation $f(\boldsymbol{x})=2 \hat{J} J\left(\boldsymbol{x}-\boldsymbol{e}_{2}\right)-\boldsymbol{e}_{2}, \boldsymbol{e}_{2}=(0,1,0)$, which takes the half-space $y \leq 0$ to the unit ball $|\boldsymbol{x}| \leq 1$ and the point $\boldsymbol{e}_{2}$ to the infinity. These are the views along the $z$-axis.

\section{REFERENCES}

[1] K. Ahara and Y. Araki. Sphairahedral approach to parameterize visible three dimensional quasi-Fuchsian fractals. Proc. of the CGI (2003), 226-229.

[2] B. N. Apanasov. Conformal geometry of discrete groups and manifolds. de Gruyter Expositions in Mathematics, 32. Walter de Gruyter \& Co., Berlin, 2000. MR1800993 (2002k:57087)

[3] A. Basmajian. Tubular neighborhoods of totally geodesic hypersurfaces in hyperbolic manifolds. Invent. Math. 117 (1994), no. 2, 207-225. MR.1273264 (95c:57020)

[4] A. F. Beardon. The geometry of discrete groups. Corrected reprint of the 1983 original. Graduate Texts in Mathematics, 91. Springer-Verlag, New York, 1995. MR1393195 (97d:22011)

[5] W. Cao, J. Parker and X. Wang. On the classification of quaternionic Möbius transformations. Mathematical Proceedings of the Cambridge Philosophical Society 137 (2004) 349-361. MR2092064 (2005f:30082)

[6] D. B. A. Epstein and C. Petronio. An exposition of Poincare's polyhedron theorem. Enseign. Math. (2) 40 (1994), no. 1-2, 113-170. MR1279064 (95f:57030) 
[7] M. Kapovich. Hyperbolic manifolds and discrete groups. Progress in Mathematics, 183. Birkhauser Boston, Inc., Boston, MA, 2001. MR.1792613 (2002m:57018)

[8] M. Kapovich. Kleinian groups in higher dimensions. In "Geometry and Dynamics of Groups and Spaces. In memory of Alexander Reznikov", M. Kapranov et al (eds). Birkhauser, Progress in Mathematics, Vol. 265, 2007, p. 485-562. MR2402415

[9] I. Kra. Horocyclic coordinates for Riemann surfaces and moduli spaces. I. Teichmuller and Riemann spaces of Kleinian groups. J. Amer. Math. Soc. 3 (1990), no. 3, 499-578. MR.1049503 (91c:32014)

[10] L. Keen and C. Series. Pleating coordinates for the Maskit embedding of the Teichmüller space of punctured tori. Topology 32 (1993), no. 4, 719-749. MR1241870 (95g:32030)

[11] A. Marden. Outer Circles. An Introduction to Hyperbolic 3-Manifolds. Cambridge University Press. New York, 2007. MR,2355387 (2008i:57001)

[12] B. Maskit. Kleinian groups. Grundlehren der Mathematischen Wissenschaften, 287, Springer-Verlag, Berlin, 1988. MR959135 (90a:30132)

[13] S. Matsumoto. Foundations of flat conformal structure. Aspects of low-dimensional manifolds, 167-261, Adv. Stud. Pure Math., 20, Kinokuniya, Tokyo, 1992. MR1208312 (93m:57014)

[14] Y. N. Minsky. The classification of punctured-torus groups. Ann. of Math. (2) 149 (1999), no. 2, 559-626. MR1689341 (2000f:30028)

[15] D. Mumford, C. Series and D. Wright. Indra's pearls. The vision of Felix Klein. Cambridge University Press, New York, 2002. MR.1913879 (2003f:00005)

Synclore Corporation, Hakuyo Building, 3-10 Nibancho Chiyoda-ku, Tokyo 102-0084, JAPAN

E-mail address: yoshiaki.araki@synclore.com

Graduate School of Mathematics, Nagoya University, Nagoya 464-8602, Japan

E-mail address: itoken@math.nagoya-u.ac.jp 